\title{
MONODROMY GROUPS AND POINCARÉ SERIES
}

BY DENNIS A. HEJHAL

1. Introduction. The early work of Klein [28] and Poincare [53] on the uniformization of algebraic curves (compact Riemann surfaces) was based on the so-called continuity method. After a few years, however, some serious objections were raised regarding both of these papers. Cf. [11, pp. 408-414, 438], [29, pp. 731-741], and [53, pp. 233-236].

Partly for this reason, mathematicians sought to develop a more direct approach to the uniformization theorem. Such an approach was finally carried out by Koebe [32] and Poincare [56] in 1907. Suffice it to say that the direct approach is based on potential theory, not on the continuity method. Some additional references are as follows: [27, pp. 73, 323], [33], [52], [69, pp. 159-179].

Although the continuity method ran into trouble (and was later abandoned), the underlying idea is still very tempting. Poincare's approach [53] is of particular interest here, because of its connection with ordinary differential equations.

When viewed in its most primitive form, the inter-relationship between conformal mapping and differential equations certainly goes back to Riemann [59]. Compare: [28, pp. 214-216] and [64]. These techniques were first applied to uniformization problems around 1880, when Poincaré [53] showed that the differential equations characterizing uniformization depend upon $3 g-3$ complex parameters. Cf. equation (10) below. If these parameters could be determined, it would then be possible to compute the desired uniformization explicitly. Unfortunately, these parameters (known in the literature as accessory parameters) are notoriously difficult to get hold of. In fact, part of the difficulty with the original continuity method arose from trying to understand what happens to these parameters when the Riemann surface degenerates; see [53, §§8-14]. Cf. also [6], [29, pp. 731-741, 774], [41], and [61, pp. 215-304].

Since no one has succeeded in writing down explicit formulas for the accessory parameters, it seems perfectly reasonable to compromise and try to obtain variational formulas instead. The obvious hope is that such formulas will offer some insight into what is going on. Part 1 will be devoted to this problem.

At first glance, our results would seem to be rather disappointing; the variational equations are so messy that they appear to be useless. However, after staring at the formulas for a few minutes, one discovers some very

This is an expanded version of an invited address delivered at the 83rd Annual Meeting of the American Mathematical Society in St. Louis, Missouri on January 29, 1977; received by the editors May 25, 1977.

AMS (MOS) subject classifications (1970). Primary 10D15, 20H10, 30A46, 30A52, 30A58, 32G15, 34A20. 
curious integrals (involving quadratic differentials). These integrals lead to a new technique for studying Poincaré series.

This technique is surprisingly effective when it comes to testing whether or not a given Poincaré series is identically zero. In fact, under certain conditions, we are able to reduce this well-known problem to a finite computation. Cf. [51, p. 249]. A brief survey of our results (in this ongoing investigation) will be given in Part 2.

\section{PART ONE}

2. The basic differential equations. The results described in this and the next two sections are taken from [18], [21]. To begin with, let $F$ be a compact Riemann surface having genus $g \geqslant 2$. We can represent $F$ as an algebraic curve $\{P(x, y)=0\}$; the polynomial $P(x, y)$ is naturally assumed to be irreducible. By considering $y$ as a function of $x$, we are able to interpret $F$ as a ramified covering of the Riemann sphere $\hat{\mathbf{C}}$.

Let $F^{\infty}$ denote the universal covering surface of $F$ (in the sense of topology). The classical uniformization theorem guarantees that $F^{\infty}$ is conformally equivalent to the upper half-plane $H$. Cf. $[2$, p. 181] and [69, p. 171]. We can therefore form the universal covering map $\varphi: H \rightarrow F$.

Let $\Gamma$ denote the associated group of cover transformations [2, pp. 37-38]:

$$
\Gamma=\{\text { self-mappings } T: \varphi(T z)=\varphi(z) \text { for all } z \in H\} .
$$

We recall that there is a well-known isomorphism between $\Gamma$ and the fundamental group $\pi_{1}(F, \theta)$. Using equation (1), we immediately see that $\Gamma$ is a Fuchsian group [i.e. a discrete subgroup of $\operatorname{PSL}(2, \mathbf{R})]$. By definition,

$$
\begin{aligned}
\operatorname{PSL}(2, \mathbf{R}) & =\{\text { the group of Möbius transformations over } \mathbf{R}\} \\
& =S L(2, \mathbf{R}) /( \pm I) .
\end{aligned}
$$

It is a theorem that $\Gamma$ can be faithfully realized as a discrete subgroup of $S L(2, \mathbf{R})$; cf. [18, pp. 217-218, 245]. This realization will be used throughout the rest of the paper.

Since $F$ is compact, $\Gamma$ has a compact fundamental polygon $\mathscr{F}$. The family $\{L(\mathscr{F}): L \in \Gamma\}$ will then form a tessellation of $H$; note that the matrices in $\Gamma-\{I\}$ are strictly hyperbolic. By identifying the sides of $\mathscr{F}$, we can now represent $F$ as a quotient space $H / \Gamma$. In other words:

$$
F=H / \Gamma \text {. }
$$

For obvious reasons, we would like to determine the covering map $\varphi$ as explicitly as possible. To do so, it is enough to study $w=\varphi^{-1}$. The function $\varphi^{-1}$ lives on $F$, but is not single-valued. In fact, under the action of $\pi_{1}(F, \theta)$, we find that:

$$
w \rightarrow L(w) \text { with } L \in \Gamma \text {. }
$$

Since $L$ is a Möbius transformation, it is natural to consider the Schwarzian derivative of $w$. But, as is easily seen, $\{w, x\}$ is a single-valued meromorphic function on $F$. Consequently:

$$
\{w, x\}=2 R(x, y) \text { for some rational function } R \text {. }
$$

The Schwarzian derivative is of course nonlinear. To linearize equation (5), 
we apply a well-known change of variable:

$$
u=\frac{1}{\sqrt{d w / d x}}, \quad v=\frac{w}{\sqrt{d w / d x}}, \quad w=\frac{v}{u} .
$$

The functions $u$ and $v$ will now satisfy

$$
d^{2} u / d x^{2}+R(x, y) u=0 .
$$

We must try to find $R(x, y)$.

To cut down on the number of possibilities, we can obviously limit ourselves to those cases where $w=v / u$ is locally $1-1$. This restriction leads to the decomposition

$$
R(x, y)=R_{0}(x, y)+Q(x, y)
$$

where $R_{0}$ is some explicit function on $F$ and $Q d x^{2}$ is a regular quadratic differential. Cf. [17], [18], and [63, pp. 265-272]. Loosely speaking, $R_{0}$ is just the second derivative of an Abelian integral of the third kind. For notational convenience, we let $\mathbb{Q}_{n}(F)$ denote the set of regular $n$th order differentials on $F$. It follows from the Riemann-Roch theorem that:

$$
\operatorname{dim}_{\mathbf{C}} \mathbb{Q}_{n}(F)=(2 n-1)(g-1) \text { for } n \geqslant 2 \text {. }
$$

Cf. [26, p. 507]. Taking $n=2$, we immediately obtain:

$$
R(x, y)=R_{0}(x, y)+\sum_{j=1}^{3 g-3} \lambda_{j} Q_{j}(x, y),
$$

where $\lambda_{j} \in \mathbf{C}$ and $\left\{Q_{j} d x^{2}\right\}_{j=1}^{3 g-3}$ is some basis for $\mathbb{Q}_{2}(F)$. Equation (7) can now be rewritten as follows:

$$
\frac{d^{2} u}{d x^{2}}+\left[R_{0}(x, y)+\sum_{j=1}^{3 g-3} \lambda_{j} Q_{j}(x, y)\right] u=0 .
$$

In some sense, the computation of $\varphi$ has been reduced to solving for the accessory parameters $\lambda_{j}$. Compare: [3, pp. 272-379], [11, pp. 233-240], [17, pp. 9-10], [18, pp. 215-216], [30], [31], [53], [59], [62], [63].

As mentioned in $\S 1$, it seems rather difficult to get hold of the $\lambda_{j}$ which give rise to the Fuchsian uniformization $\varphi$. To avoid possible confusion, we shall call these special values of $\lambda_{j}$ the Fuchsian parameters. [These parameters should be regarded as functions of the underlying Riemann surface $F$.]

Later on, it will be necessary to consider certain vector spaces over $\mathbf{R}$ instead of $\mathbf{C}$. For this reason, we shall use real accessory parameters $\lambda_{j}$ in a slightly modified differential equation:

$$
\frac{d^{2} u}{d x^{2}}+\left[R_{0}(x, y)+\sum_{j=1}^{6 g-6} \lambda_{j} Q_{j}(x, y)\right] u=0 ;
$$

the quadratic differentials $Q_{j}(x, y) d x^{2}$ correspond to a basis over $\mathbf{R}$.

Consider equation (11) with arbitrary $\lambda_{j} \in \mathbf{R}$; let $u$ and $v$ be any two linearly independent solutions. The quotient $z=v / u$ will then be a linearly polymorphic function on $F$. More precisely, after travelling around the loop 
$M \in \pi_{1}(F, \mathcal{O})$, we immediately see that:

$$
\left(\begin{array}{l}
v \\
u
\end{array}\right) \rightarrow \hat{M}\left(\begin{array}{l}
v \\
u
\end{array}\right) \text { with } \hat{M} \in S L(2, \mathrm{C})
$$

The mapping $M \rightarrow \hat{M}$ is called the monodromy homomorphism; the image set $\{\hat{M}\}$ is called the monodromy group.

When the Fuchsian parameters are substituted for $\lambda_{j}$, the monodromy group obviously reduces to $\Gamma$ (apart from a possible conjugation). This fact suggests that variational equations for the Fuchsian parameters can be formulated by writing down the condition that $\{\hat{M}\}$ is conjugate to a subgroup of $S L(2, \mathbf{R})$. The extra conjugation causes a technical problem which can be eliminated by working with the (seemingly) weaker condition that $\operatorname{Tr}(\hat{M})$ be real.

REMARK. There exist non-Fuchsian parameters $\lambda_{j}$ for which $\{\hat{M}\}$ looks just like a Fuchsian group. This phenomenon is explained in [17] and can always be avoided by working locally. Since variational equations are local by definition, there is no need to worry about pathologies of this type.

3. The variational formulas. In order to obtain variational equations for the Fuchsian parameters, it is very tempting to work with the condition that $\operatorname{Tr}\left(\hat{M}_{k}\right)$ be real for enough $M_{k}$. The underlying idea here is rather obvious: if enough traces are real, then $\{\hat{M}\}$ should be conjugate to a subgroup of $S L(2, \mathbf{R})$. Under these circumstances, the local results in [17] will guarantee that the accessory parameters are really Fuchsian parameters.

To illustrate the necessary computations, it is sufficient to consider a closed curve $M \subseteq \mathbf{C}$. Let $x_{0}$ be its initial point; suppose that $E_{0}$ and $Q_{0}$ are holomorphic functions near $M$. We want to study the following differential equation for small values of $\varepsilon$ :

$$
\left\{\begin{array}{c}
d^{2} u / d x^{2}+\left[E_{0}+\varepsilon Q_{0}\right] u=0 \\
u\left(x_{0}\right)=1, \quad v\left(x_{0}\right)=0 \\
u^{\prime}\left(x_{0}\right)=0, \quad v^{\prime}\left(x_{0}\right)=1
\end{array}\right\} .
$$

Let $\left(\begin{array}{c}v_{a} \\ v_{k}\end{array}\right)$ denote the obvious solution vector [defined by analytic continuation along $M$ ]; use equation (12) to define the corresponding monodromy matrix $\hat{M}(\varepsilon)$. By means of a straightforward perturbation expansion, we find that:

$$
\begin{aligned}
& \left(\begin{array}{l}
v_{\varepsilon} \\
u_{\varepsilon}
\end{array}\right)=\left[I+\varepsilon \int_{x_{0}}^{x} Q_{0}\left(\begin{array}{ll}
-u v & v^{2} \\
-u^{2} & u v
\end{array}\right) d t\right]\left(\begin{array}{l}
v \\
u
\end{array}\right)+O\left(\varepsilon^{2}\right) ; \\
& \hat{M}(\varepsilon)=\left[I+\varepsilon \oint_{M} Q_{0}\left(\begin{array}{ll}
-u v & v^{2} \\
-u^{2} & u v
\end{array}\right) d t\right] \hat{M}+O\left(\varepsilon^{2}\right) .
\end{aligned}
$$

As an abbreviation, we have written

$$
\left(\begin{array}{l}
v \\
u
\end{array}\right)=\left(\begin{array}{l}
v_{0} \\
u_{0}
\end{array}\right) \text { and } \hat{M}=\hat{M}(0)
$$

For later use, we define:

$$
\Re(u ; v)=\left(\begin{array}{cc}
-u v & v^{2} \\
-u^{2} & u v
\end{array}\right) .
$$


There are obvious analogs of equations (14) and (15) on the surface $F$ provided that $M$ stays away from the branch points.

Using equation (15), it is now very simple to formulate some (necessary) variational conditions for the Fuchsian parameters. To begin with, let $\{F(b):-\delta<b<\delta\}$ be any $C^{\infty}$ family of compact Riemann surfaces $\{P(x, y, b)=0\}$ passing through $F(0)=F$. The number $\delta$ is assumed to be very small. The functions $R_{0}$ and $Q_{j}$ in equation (11) can then be chosen so as to be $C^{\infty}$ with respect to $b$. With a suitable interpretation, the same can be said about the branch points. To obtain the desired variational conditions, we merely combine the trace of equation (15) with the obvious restriction:

$$
\partial \operatorname{Im} \operatorname{Tr}\left(\hat{M}_{k}\right) / \partial b=0 .
$$

THEOREM 1. Let $f_{j}(b)$ denote the Fuchsian parameters for $F(b)$. Then:

$$
\begin{aligned}
\sum_{j=1}^{6 g-6} \frac{\partial f_{j}}{\partial b} \operatorname{Im} \oint_{M_{k}} Q_{j} \operatorname{Tr}\left[\Re(u ; v) \hat{M}_{k}\right] d x \\
=-\operatorname{Im} \oint_{M_{k}}\left[\frac{\partial R_{0}}{\partial b}+\sum_{j=1}^{6 g-6} f_{j} \frac{\partial Q_{j}}{\partial b}\right] \operatorname{Tr}\left[\Re(u ; v) \hat{M}_{k}\right] d x,
\end{aligned}
$$

where the derivatives are evaluated at $b=0$ and $\left(\begin{array}{c}v \\ u\end{array}\right)$ corresponds to the Fuchsian uniformization of $F$. The paths $M_{k} \in \pi_{1}(F, \theta)$ are completely arbitrary.

Since the solution vector $\left(\begin{array}{l}v \\ u\end{array}\right)$ depends upon $f_{j}$, this system of equations is quasi-linear. To solve for the derivatives $\partial f_{j} / \partial b$, one must try to select $6 g-6$ paths $M_{k}$ so that the functions $\operatorname{Tr}\left(\hat{M}_{k}\right)$ are [in some sense] independent.

To make this selection process more precise, we proceed as follows. First of all, choose a canonical dissection $\left\{\alpha_{k}, \beta_{k}\right\}_{k=1}^{g}$ for $F$; see [65, p. 113]. The fundamental group $\pi_{1}(F, \mathcal{\theta})$ is freely generated by these paths except for the commutator relation

$$
\left(\alpha_{1}, \beta_{1}\right) \cdots\left(\alpha_{g}, \beta_{g}\right)=I \text {. }
$$

Applying the isomorphism $\pi_{1}(F, \theta) \cong \Gamma$ and the monodromy homomorphism, we immediately obtain:

$$
\begin{aligned}
\pi_{1}(F, \mathcal{\theta}) & =\left[\alpha_{1}, \ldots, \alpha_{g} ; \beta_{1}, \ldots, \beta_{g}\right] ; \\
\Gamma & =\left[A_{1}, \ldots, A_{g} ; B_{1}, \ldots, B_{g}\right] ; \\
\text { monodromy group } & =\left[X_{1}, \ldots, X_{g} ; Y_{1}, \ldots, Y_{g}\right] .
\end{aligned}
$$

In each case, the obvious commutator relation holds. The groups (19)-(21) are now said to be marked. Compare: [17, pp. 14-19].

The marked monodromy groups obviously belong to the following algebraic variety:

$$
N=\left\{\left[X_{\alpha} ; Y_{\beta}\right] \in S L(2, \mathrm{C})^{2 g}:\left(X_{1}, Y_{1}\right) \cdots\left(X_{g}, Y_{g}\right)=I\right\} .
$$

By counting the constants, we immediately see that $\operatorname{dim}_{\mathrm{C}}(N)=6 g-3$. Since the monodromy group of equation (11) is determined only up to a conjugation, it is natural to consider the quotient variety 


$$
N / S L(2, \mathrm{C})=N[\text { modulo inner automorphisms }] .
$$

This variety has complex dimension $6 g-6$.

The varieties $N$ and $N / S L(2, \mathrm{C})$ have been studied by Teichmüller [66] and Gunning [13], [14]. Both varieties are known to be nonsingular near the Fuchsian group $\Gamma$.

Return to equations (19) and (21) for a minute. Under the monodromy homomorphism, each element of $\pi_{1}(F, \theta)$ corresponds to a word involving the letters $\left\{X_{k}, Y_{k}\right\}_{k=1}^{g}$. This lexicographic correspondence will be denoted by $M \rightarrow \tilde{M}$. Cf. equation (12). It is rather obvious that $\operatorname{Tr}(\tilde{M})$ is a single-valued holomorphic function on $N / S L(2, \mathrm{C})$.

FACT (near $\Gamma$ ). One can always select paths $\left\{M_{k}\right\}_{k=1}^{6 g-6}$ so that the functions $\operatorname{Tr}\left(\tilde{M}_{k}\right)$ are local coordinates on $N / S L(2, \mathbf{C})$. In this context, Fuchsian groups are characterized by the condition $\left\{\operatorname{Im} \operatorname{Tr}\left(\tilde{M}_{k}\right)=0\right\}$.

Knowledge of $\operatorname{Tr}\left(\tilde{M}_{k}\right)$ will thus determine $\left[X_{\alpha} ; Y_{\beta}\right]$ up to conjugation. Since Fricke [10, pp.284-398] has used similar traces to provide a global characterization of Fuchsian groups, the $M_{k}$ will be called Fricke paths. These paths are written down quite explicitly in [18, p. 247]. For $g=2$ and generic $\Gamma$, we have:

$$
\left\{\begin{array}{l}
M_{1}=\alpha_{1}, M_{2}=\beta_{1}, M_{3}=\alpha_{2}, \quad M_{4}=\beta_{2}, M_{5}=\left(\alpha_{1}, \beta_{1}\right), M_{6}=\alpha_{1} \alpha_{2} \\
\tilde{M}_{1}=X_{1}, \tilde{M}_{2}=Y_{1}, \tilde{M}_{3}=X_{2}, \tilde{M}_{4}=Y_{2}, \tilde{M}_{5}=\left(X_{1}, Y_{1}\right), \tilde{M}_{6}=X_{1} X_{2}
\end{array}\right\} .
$$

Compare: equation (66).

THEOREM 2. Let $\left\{M_{k}\right\}_{k=1}^{6 g-6}$ be the Fricke paths. The coefficient matrix

$$
\left[\operatorname{Im} \oint_{M_{k}} Q_{j} \operatorname{Tr}\left[\Re(u ; v) \hat{M}_{k}\right] d x\right]_{j k}
$$

which appears in Theorem 1 will then have maximal rank $6 g-6$.

This theorem is proved in [18, p. 255] using the general theory of monodromy groups. Roughly speaking, the idea of the proof is as follows. If the rank were less than maximal, the quasi-linear equations in Theorem 1 could be solved to obtain two distinct families of differential equations (11), both characterized by (17). Since the corresponding Fricke coordinates $\operatorname{Tr}\left(\hat{M}_{k}\right)$ are real, these differential equations will yield two distinct Fuchsian uniformizations for certain $F(b)$. This is a contradiction.

Notice that Theorem 2 permits us to solve for $\partial f_{j} / \partial b$ [in Theorem 1]. The resulting formulas are obviously very messy and appear to be quite useless.

4. Another look at the variational equations. Before giving up on those formulas for $\partial f_{j} / \partial b$, it makes sense to ask: what is the significance of the coefficient matrix in Theorem 2?

In this way, we are led to consider the integrals:

$$
\oint_{M} Q \operatorname{Tr}\left[\left(\begin{array}{cc}
-u v & v^{2} \\
-u^{2} & u v
\end{array}\right) \hat{M}\right] d x \quad \text { for } M \in \pi_{1}(F, \theta) .
$$


These periods are hard to interpret [on $F$ ] because they involve the Fuchsian uniformization $z=v / u$. But, remember that $F=H / \Gamma$. When computed on $H$, the above integral becomes:

$$
\int_{z_{0}}^{M z_{0}} q(z) \operatorname{Tr}\left[\left(\begin{array}{cc}
-z & z^{2} \\
-1 & z
\end{array}\right) M\right] d z
$$

where $Q d x^{2}=q(z) d z^{2}$ and $M \in \Gamma$. Cf. equation (6) and recall that $\Gamma \cong$ $\pi_{1}(F, \vartheta)$. It should also be noted here that $q(z) d z^{2}$ is a regular quadratic differential on $H / \Gamma$.

The periods defined by (26) seem rather curious. They obviously live on $H / \Gamma$. But what do they mean? And what are their properties?

To start off with, we define:

$$
\pi(z)=\left(\begin{array}{cc}
-z & z^{2} \\
-1 & z
\end{array}\right)
$$

For any matrix $T \in S L(2, \mathrm{C})$, it is easily verified that:

$$
\Re(T z)=T^{\prime}(z) \cdot T \Re(z) T^{-1} \text {. }
$$

This trivial identity will turn out to be quite important. For example, by using (28) and the fact that $q(K z) K^{\prime}(z)^{2}=q(z)$ for $K \in \Gamma$, we immediately see that:

$$
\left\{\begin{array}{l}
(26) \text { is independent of } z_{0}, \\
\left.(26) \text { is unchanged when } M \text { is replaced by } K M K^{-1} \text { (with } K \in \Gamma\right)
\end{array}\right\} \text {. }
$$

The identity $q(K z) K^{\prime}(z)^{2}=q(z)$ simply reflects the fact that $Q d x^{2}=q(z) d z^{2}$ $=q(K z) d(K z)^{2}$.

Since (26) is a scalar, we are not dealing with an Eichler period. Cf. [9] and equations (104)-(106).

Fortunately, it is not difficult to find a variational interpretation for (26). To do so, we consider the differential equation

$$
d^{2} u / d z^{2}+\varepsilon q(z) u=0 \text { on } H / \Gamma
$$

subject to

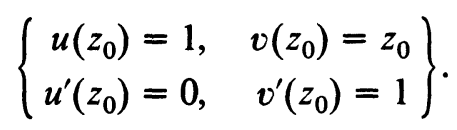

Let

$$
\mathscr{U}_{\varepsilon}(z)=\left(\begin{array}{l}
v_{\varepsilon} \\
u_{\varepsilon}
\end{array}\right)
$$

be the obvious solution vector; by construction $\mathscr{Q}_{0}(z)=\left(\begin{array}{l}z \\ 1\end{array}\right)$. For $M=\left(\begin{array}{ll}a & b \\ c & d\end{array}\right) \in$ $\Gamma$, we easily see that:

$$
\mathcal{Q}_{\varepsilon}(M z)=\hat{M}(\varepsilon) \mathcal{Q}_{\varepsilon}(z) \cdot(c z+d)^{-1} \text { with } \hat{M}(\varepsilon) \in S L(2, \mathbf{C}) .
$$

Compare [23]; equation (32) is an obvious analog of (12). Using a perturbation expansion as in (14)-(15), we discover that [18, p. 223]: 


$$
\begin{gathered}
{\left[\frac{\partial \hat{M}(\varepsilon)}{\partial \varepsilon}\right]_{\varepsilon=0}=\int_{z_{0}}^{M z_{0}} q(z) \operatorname{TL}(z) M d z ;} \\
{\left[\frac{\partial}{\partial \varepsilon} \operatorname{Tr} \hat{M}(\varepsilon)\right]_{\varepsilon=0}=\int_{z_{0}}^{M z_{0}} q(z) \operatorname{Tr}[\operatorname{TL}(z) M] d z .}
\end{gathered}
$$

Equation (34) makes fact (29) seem rather obvious. More importantly, however, the coefficient matrix in Theorem 2 can now be regarded as a Jacobian matrix: we merely take $u^{\prime \prime}+\left[\Sigma \lambda_{j} q_{j}(z)\right] u=0$ and look at the equidimensional mapping $\left(\lambda_{j}\right) \rightarrow\left(\operatorname{Im} \operatorname{Tr}\left(\hat{M}_{k}\right)\right)$. Equations (33)-(34) will reappear later in a more general context [cf. Theorems 8 and 11].

Finally, observe that Theorem 2 can be reformulated as follows.

THEOREM 3. Given any regular quadratic differential $Q(z) d z^{2}$ on $H / \Gamma$. Then: $Q(z) \equiv 0$ if and only if

$$
\operatorname{Im} \int_{z_{0}}^{M_{k} z_{0}} Q(z) \operatorname{Tr}\left[\mathscr{T L}(z) M_{k}\right] d z=0
$$

for $1 \leqslant k \leqslant 6 g-6$. The elements $M_{k}$ in $\Gamma$ correspond to the Fricke paths.

\section{PART TWO}

5. Poincaré series. When looking for possible applications of Theorem 3 , it seems natural to consider those regular quadratic differentials $Q(z) d z^{2}$ which can be written as Poincaré series; i.e.

$$
Q(z)=\theta[z, R]=\sum_{T \in \Gamma} R(T z) T^{\prime}(z)^{2} .
$$

[We refer to equations (40)-(41) for a more careful description.]

Motivated by my earlier work with Schottky groups [19], [20], I immediately asked myself if Theorem 3 could be used to test whether $\theta[z, R] \equiv 0$ on $H$. This possibility will be examined in $\$ 6$; compare [21], [22]. The corresponding results for $n$th order differentials will be given in $\$ 7$.

To set the stage, we need a few preliminaries. First of all, fix any integer $n \geqslant 2$ and recall that $F=H / \Gamma$. The concept of an $n$th order differential on the Riemann surface $F$ is well known and needs no further explanation [cf. $Q_{n}(F)$ in $\left.\S 2\right]$. When these $n$th order differentials are transferred over to $H$, they become automorphic forms of weight $n$. In particular, the automorphic forms corresponding to regular $n$th order differentials comprise a family

$$
Q_{n}(H, \Gamma)=\left\{\begin{array}{ll}
\varphi: & \varphi \text { is holomorphic on } H \\
& \varphi(T z) T^{\prime}(z)^{n}=\varphi(z) \text { for } T \in \Gamma \text { and } z \in H
\end{array}\right\}
$$

Since the Fuchsian group $\Gamma$ also acts discontinuously on the lower half-plane $L$, we define:

$$
\begin{gathered}
D=H \cup L \\
\mathbb{Q}_{n}(D, \Gamma)=\left\{\begin{array}{l}
\varphi \text { is holomorphic on } D \\
\varphi(T z) T^{\prime}(z)^{n}=\varphi(z) \text { for } T \in \Gamma \text { and } z \in D
\end{array}\right\} .
\end{gathered}
$$

The vector space $\mathcal{Q}_{n}(D, \Gamma)$ can obviously be written as a direct sum: 


$$
\mathbb{Q}_{n}(D, \Gamma)=\mathbb{Q}_{n}(H, \Gamma) \oplus \mathbb{Q}_{n}(L, \Gamma) .
$$

Using equation (9), we immediately see that:

$$
\operatorname{dim}_{\mathbf{C}} \mathbb{Q}_{n}(H, \Gamma)=\operatorname{dim}_{\mathbf{C}} \mathbb{Q}_{n}(F)=(2 n-1)(g-1) .
$$

Compare: [34, pp. 328-330].

We can now introduce the Poincaré series of weight $n$ [51]. Let $\hat{\mathbf{R}}$ denote the extended real axis and

(40) $\Re_{n}=\left\{\begin{array}{ll}R: & \begin{array}{l}R(z) \text { is a rational function, the poles of } R(z) d z^{n} \text { are } \\ \text { situated along } \hat{\mathbf{R}} \text { and have multiplicity }<n-1\end{array}\end{array}\right\}$.

For each $R \in \Re_{n}$, we define a Poincaré series $\theta[z, R]$ as follows:

$$
\theta[z, R]=\sum_{T \in \Gamma} R(T z) T^{\prime}(z)^{n} .
$$

By means of a formal computation, we immediately see that $\theta[z, R] \in$ $\mathbb{Q}_{n}(D, \Gamma)$. This assertion is easily justified, because the definition of $\Re_{n}$ guarantees that $\theta[z, R]$ is absolutely convergent on $D$; see [34, pp. 91, 99, 113]. On the other hand, if we restrict ourselves to $\mathbb{Q}_{n}(H, \Gamma)$, then (40) can obviously be modified to allow $R(z)$ to have poles in $L$.

Many people have studied the problem of trying to represent arbitrary elements of $\mathscr{Q}_{n}(H, \Gamma)$ as Poincaré series $\theta\left[z, R_{0}\right]$. The earliest work in this area is due to Poincaré himself [51]. He proved that such representations are possible, at least when $R_{0}(z)$ is allowed to have poles in $L$. Petersson [47], [48] later clarified and extended this work by using his inner product:

$$
\left(f_{1}, f_{2}\right)=\iint_{\mathscr{F}} f_{1}(z) \overline{f_{2}(z)} y^{2 n-2} d x d y .
$$

Loosely speaking, the crucial idea in Petersson's work is the construction of certain Poincaré series $E_{m}$ such that:

$$
\left(f, E_{m}\right)=f^{(m)}\left(z_{0}\right) \text { for all } f \in \mathbb{Q}_{n}(H, \Gamma) .
$$

Automorphic forms $f$ in the orthogonal complement of $\left\{E_{0}, E_{1}, \ldots, E_{N}\right\}$ must therefore possess a zero having multiplicity at least $N+1$. Since the total number of zeros cannot exceed $2 n(g-1)$ [when $f \neq 0$ ], we immediately see that $\left\{E_{0}, E_{1}, \ldots, E_{N}\right\}$ spans $\mathbb{Q}_{n}(H, \Gamma)$ whenever $N \geqslant 2 n(g-1)$. We also note that:

$$
2 n(g-1)+1=\operatorname{dim}_{\mathbf{C}} \mathbb{Q}_{n}(H, \Gamma)+g .
$$

To obtain representations of the form $\theta[z, R]$ with $R \in \mathcal{R}_{n}$, one can now apply an approximation argument. See [5] and [34, pp. 91, 130-141].

The analogous problem for $D$ was first studied by Bers around 10 years ago in the context of Kleinian groups; see [34, pp. 186, 383]. Bers proved that any element of $\mathbb{Q}_{n}(D, \Gamma)$ can be represented in the form $\theta[z, R]$ with $R \in \Re_{n}$. The fact that the same $R$ can be used for both $H$ and $L$ is not too surprising in view of certain well-known theorems about rational approximation [68, p. 15].

The results considered above are examples of completeness theorems. Such 
theorems do not tell the "complete" story about Poincare series [even if they do provide a semi-explicit basis for $\mathbb{Q}_{n}(H, \Gamma)$ or $\left.\mathbb{Q}_{n}(D, \Gamma)\right]$. For example, consider $\mathbb{Q}_{n}(D, \Gamma)$. Since

$$
\operatorname{dim}_{\mathbf{C}}\left(\Re_{n}\right)=\infty \text { and } \operatorname{dim}_{\mathbf{C}} \mathbb{Q}_{n}(D, \Gamma)=2(2 n-1)(g-1),
$$

Bers' result shows that there must exist many rational functions $R \in \Re_{n}$ for which $\theta[z, R] \equiv 0$ (on $D$ ). Completeness theorems offer very little insight into this situation. We know that such $R$ exist, but we cannot write them down explicitly.

The problem of finding (explicit) necessary/sufficient conditions for $\theta[z, R] \equiv 0$ was first raised by Poincare $[51$, p. 249]. This problem can be posed for both $\mathbb{Q}_{n}(H, \Gamma)$ and $\mathbb{Q}_{n}(D, \Gamma)$. For the case $\mathbb{Q}_{n}(H, \Gamma)$, a few very partial results are known; see [51, pp. 249-254] and [45].

TRIVIAL REMARK. We observe that $\theta[z, R] \equiv 0$ on $D$ whenever $R(z)=$ $A(z)-A(L z) L^{\prime}(z)^{n}, A \in \Re_{n}, L \in \Gamma$. This fact was first noted by Poincaré [51, p. 249].

We claim that there is good reason to believe that life is extra complicated when everything is restricted to $H$. For simplicity, suppose that $n=2$. Choose any nonzero $\varphi \in \mathbb{Q}_{2}(L, \Gamma)$. According to Bers' completeness theorem, there will now exist some $R \in \Re_{2}$ such that

$$
\theta[z, R]=\left\{\begin{array}{ll}
0 & \text { for } z \in H \\
\varphi(z) & \text { for } z \in L
\end{array}\right\} .
$$

To see things more clearly, we decompose $R(z)$ using partial fractions:

$$
R(z)=\sum_{j=1}^{N} \frac{c_{j}}{z-\xi_{j}} \quad\left[c_{j} \in \mathbf{C}, \xi_{j} \in \mathbf{R}\right] .
$$

We also define $\varphi^{*}(z)=\overline{\varphi(\bar{z})}$ and $R^{*}(z)=\overline{R(\bar{z})}$. Since $\Gamma$ is a discrete subgroup of $S L(2, \mathbf{R})$, it is easy to see that

$$
\theta\left[z, R^{*}\right]=\left\{\begin{array}{ll}
\varphi^{*}(z) & \text { for } z \in H \\
0 & \text { for } z \in L
\end{array}\right\} .
$$

Thus, for $z \in H$, we find that $\theta[z, R] \equiv 0$ although $\theta\left[z, R^{*}\right] \neq 0$. But,

$$
R^{*}(z)=\sum_{j=1}^{N} \frac{\bar{c}_{j}}{z-\xi_{j}} .
$$

Consequently, if there are necessary/sufficient conditions for testing whether $\theta[z, R] \equiv 0$ on $H$, they must somehow distinguish between $c_{j}$ and $\bar{c}_{j}$. This seems a little strange [at first sight] because the $\xi_{j}$ are real and $\Gamma \subseteq S L(2, \mathbf{R})$. To say the least, such conditions could not be given by rational expressions over the field $\mathbf{Q}\left(c_{j}\right.$, coef $\left.\Gamma, \xi_{j}\right)$. Note: coef $\Gamma$ is the set of all matrix coefficients determined by $\Gamma$. Similar remarks obviously hold for $n \geqslant 3$.

In view of this difficulty, it seems reasonable to work with $\mathbb{Q}_{n}(D, \Gamma)$ instead. Since every function $R \in \Re_{n}$ can be written in the form

$$
R=R_{1}+i R_{2} \quad \text { with }\left\{\begin{aligned}
R_{1} & \in \mathscr{R}_{n}, & R_{2} & \in \Re_{n} \\
R_{1}(\bar{z}) & =\overline{R_{1}(z)}, & & R_{2}(\bar{z})=\overline{R_{2}(z)}
\end{aligned}\right\},
$$


we immediately see that $\theta[z, R] \equiv 0$ on $D$ if and only if $\theta\left[z, R_{j}\right] \equiv 0$. There is accordingly no loss of generality if we restrict ourselves to the case

$$
R \in \Re_{n}, \quad R(\bar{z})=\overline{R(z)} .
$$

From now on, only case (49) will be considered.

\section{Results for quadratic differentials.}

THEOREM $4(n=2)$. Assume that (49) holds. In addition, suppose that the poles of $R(z) d z^{2}$ are located at hyperbolic fixpoints [of certain elements in $\Gamma$ ]:

$$
R(z)=\sum_{j=1}^{N} \frac{c_{j}}{z-\xi_{j}}, \quad c_{j} \in \mathbf{R} .
$$

Then:

$$
\operatorname{Im} \int_{z_{0}}^{M z_{0}} \theta[z, R] \operatorname{Tr}[\operatorname{T(}(z) M] d z=\sum_{j=1}^{N} c_{j} X_{j M},
$$

where $X_{j M}$ is a rational expression in the field $\mathbf{Q}\left(\operatorname{coef} \Gamma, \xi_{1}, \ldots, \xi_{N}\right)$. The expressions $X_{j M}$ can be computed effectively.

Proof (sketch). By means of an auxiliary conjugation, we may assume that $\infty$ is not a pole of $R(z) d z^{2}$. Thus $R(z)=O\left(z^{-4}\right)$ near $z=\infty$. For the sake of simplicity, we assume further that the $\xi$, occur in pairs and that $M$ is primitive. [A matrix $T \in \Gamma$ is said to be primitive when it cannot be written in the form $T=U^{m}$ with $m \geqslant 2$ and $U \in \Gamma$.]

To begin the computation, observe that:

$$
\begin{aligned}
J & \stackrel{\text { def }}{=} \int_{z_{0}}^{M z_{0}} \theta[z, R] \operatorname{Tr}[\Re(z) M] d z \quad\left(z_{0} \in H\right) \\
& =\sum_{T \in \Gamma} \int_{z_{0}}^{M z_{0}} R(T z) T^{\prime}(z)^{2} \operatorname{Tr}[\Re(z) M] d z .
\end{aligned}
$$

Using the coset decomposition $\Gamma=\Sigma W[M]$, we now set $T=W M^{k}$. Therefore:

$$
J=\sum_{W, k} \int_{z_{0}}^{M z_{0}} R\left(W M^{k} z\right)\left(W M^{k}\right)^{\prime}(z)^{2} \operatorname{Tr}[\operatorname{T(}(z) M] d z .
$$

It is very tempting to let $u=M^{k} z$. Applying equation (28), we immediately obtain

$$
\Re(u)=\frac{d u}{d z} \cdot M^{k} \Re(z) M^{-k}
$$

and

$$
J=\sum_{W} \sum_{k} \int_{M^{k} z_{0}}^{M^{k+1} z_{0}} R(W u) W^{\prime}(u)^{2} \operatorname{Tr}[\operatorname{T}(u) M] d u .
$$

The summation over $k$ yields

$$
\int_{\mathfrak{Q}_{M}} R(W u) W^{\prime}(u)^{2} \operatorname{Tr}[\operatorname{TR}(u) M] d u,
$$


where $\mathbb{Q}_{M}$ is some path (in $H$ ) which connects the repulsive and attractive fixpoints of $M$; i.e. $\eta_{M}$ and $\xi_{M}$. The Cauchy integral theorem allows us to deform $\mathbb{Q}_{M}$. We can therefore redefine $\mathbb{Q}_{M}$ to be the non-Euclidean line determined by $\eta_{M}$ and $\xi_{M}$. However, we reserve the right to make some small deformations a bit later! In any case, it follows that:

$$
J=\sum_{W} \int_{\mathfrak{Q}_{M}} R(W u) W^{\prime}(u)^{2} \operatorname{Tr}[\mathfrak{T}(u) M] d u .
$$

Taking $v=W(u)$ and applying (28), we now obtain

$$
\Re(v)=\frac{d v}{d u} \cdot W \Re(u) W^{-1}
$$

and

$$
J=\sum_{W} \int_{W\left(\mathbb{E}_{M}\right)} R(v) \operatorname{Tr}\left[\Re(v) W M W^{-1}\right] d v
$$

If $\mathbb{Q}_{M}$ is chosen to be the non-Euclidean axis of $M$, then $W\left(\mathbb{Q}_{M}\right)$ will simply be the axis of $W M W^{-1}$. By using the identity

$$
\operatorname{Tr}[\operatorname{T(}(z) K]=c z^{2}+(d-a) z-b=c\left(z-\xi_{k}\right)\left(z-\eta_{k}\right)
$$

which is valid for any hyperbolic matrix $K=\left(\begin{array}{cc}a & b \\ c & d\end{array}\right) \in S L(2, \mathbf{R})$, we see that the $W\left(\mathscr{Q}_{M}\right)$ integrals are perfectly harmless.

Let $\mathfrak{Q}_{M}^{*}$ be the closed loop generated by $\mathfrak{Q}_{M}$ under the reflection principle. As is trivially verified,

$$
\operatorname{Im} \int_{\mathbb{Q}_{M}} F(z) d z=\frac{1}{2 i} \oint_{\mathbb{Q}_{M}^{*}} F(z) d z \quad \text { whenever } F(\bar{z})=\overline{F(z)} .
$$

Since $R(\bar{v})=\overline{R(v)}$, we immediately deduce that:

$$
\operatorname{Im}(J)=\sum_{W} \frac{1}{2 i} \oint_{W\left(\mathbb{Q}_{M}^{*}\right)} R(v) \operatorname{Tr}\left[\operatorname{T(}(v) W M W^{-1}\right] d v .
$$

Although each integral is elementary, the number of $W \in \Gamma /[M]$ is infinite. At first sight, this looks discouraging.

Observe, however, that the $W$ integrals are zero unless $W\left(\mathscr{Q}_{M}^{*}\right)$ separates the points $\xi_{j}$. Since this separation is considered on the Riemann sphere $\hat{\mathbf{C}}$, the estimate $R(v)=O\left(v^{-4}\right)$ plays an important role here.

Loosely speaking, there are only two kinds of separators $W\left(\mathscr{Q}_{M}^{*}\right)$ : big ones and little ones. To be more precise [and less picturesque], we let $N_{1}$ and $N_{2}$ signify the number of $\xi_{j}$ contained in the respective components of $\hat{\mathbf{C}}-$ $W\left(Q_{M}^{*}\right)$. Big separators are characterized by the condition $\min \left[N_{1}, N_{2}\right]>1$. Little separators, on the other hand, satisfy $\min \left[N_{1}, N_{2}\right]=1$. See Figure 1 .

We claim that the number of big separators $W\left(\mathfrak{Q}_{M}^{*}\right)$ is finite. To prove this assertion, notice that the endpoints of $W\left(\mathbb{Q}_{M}\right)$ are given by $W \xi_{M}, W \eta_{M}$. If $\rho$ denotes spherical distance, then $\rho\left[W \xi_{M}, W \eta_{M}\right]$ will obviously exceed some positive number $\delta_{0}$ for every big separator. However, since $\Gamma$ is discrete, a simple compactness argument [based on $W M W^{-1}$ ] shows that

$$
\operatorname{card}\left\{W \in \Gamma /[M]: \rho\left[W \xi_{M}, W \eta_{M}\right] \geqslant \delta\right\}<\infty
$$



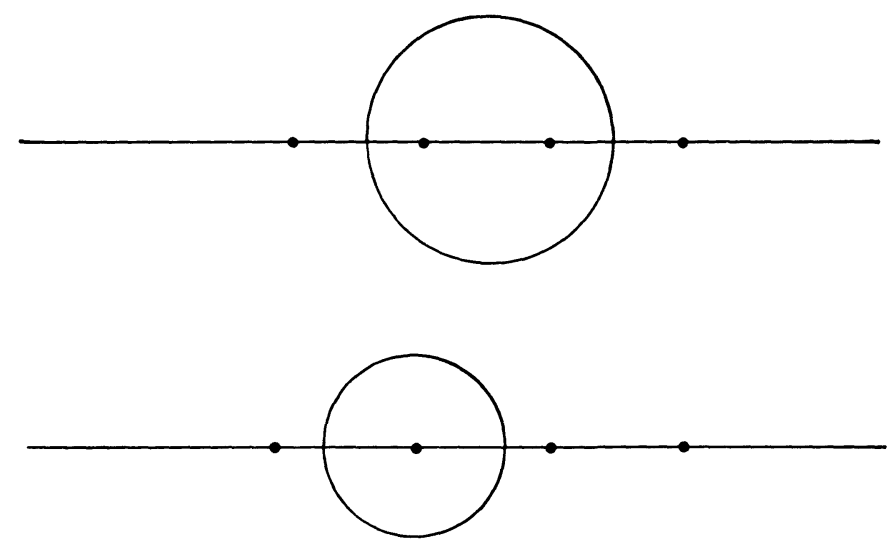

FIGURE 1

for every $\delta>0$. This, needless to say, completes the proof of our claim.

To finish the computation of $\operatorname{Im}(J)$, it is now sufficient to consider the little separators which enclose $\xi_{1}$. Since $\xi_{1}$ was assumed to be a hyperbolic fixpoint, we can write $\xi_{1}=\xi_{L}$ for some primitive $L \in \Gamma$. The repulsive fixpoint $\eta_{L}$ will then correspond to some point $\xi_{j}$ with $j \geqslant 2$.

By neglecting a finite number of elementary terms, we can reduce the problem to the case of tiny separators $W\left(\mathbb{Q}_{M}^{*}\right)$ [cf. (54)]. That is: $\rho\left[W \xi_{M}, W \eta_{M}\right]$ is now assumed to be very small.

Subject to small perturbations, $\mathbb{Q}_{M}$ was chosen earlier to be a nonEuclidean line. The same set-up can certainly be used for $\mathbb{Q}_{L}$. Since $W\left(\mathbb{Q}_{M}^{*}\right)$ is a tiny separator, it is obvious that $W\left(\mathbb{Q}_{M}\right)$ must intersect $\mathbb{Q}_{L}$. See Figure 2.

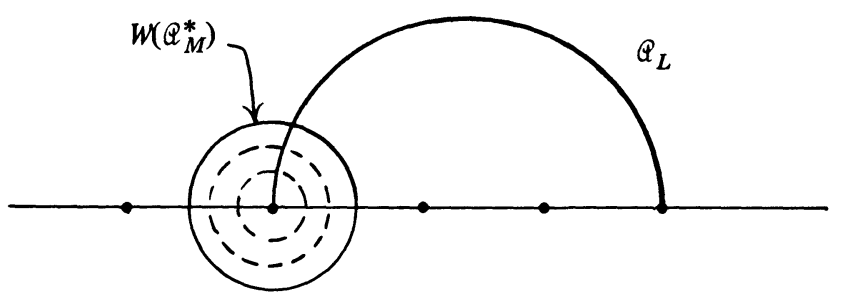

FIGURE 2

To analyze the corresponding $W \in \Gamma /[M]$, we look at $W\left(\mathbb{Q}_{M}\right) \cap \mathbb{Q}_{L}$ and pass back to $F$ using the universal covering map $\varphi$. This reduces the problem to a study of the intersections of $\gamma_{M}$ and $\gamma_{L}$ on $F$ [where $\gamma_{L}=\varphi\left(\mathbb{Q}_{L}\right)$ and $\left.\gamma_{M}=\varphi\left(\mathbb{Q}_{M}\right)\right]$. For technical reasons, it is important to know that these intersections are all transversal; this can be accomplished by making small deformations. It follows that:

$$
\begin{aligned}
\left\{W \in \Gamma /[M]: W\left(\mathbb{Q}_{M}^{*}\right) \text { is a tiny separator about } \xi_{L}\right\} \\
=\bigcup_{\alpha}\left\{L^{n} W_{\alpha}: 0 \leqslant n<\infty\right\},
\end{aligned}
$$

where $\alpha$ runs over an appropriate (but finite) index set. The dotted circles in Figure 2 should therefore be interpreted as $L W\left(\mathbb{Q}_{M}^{*}\right)$ and $L^{2} W\left(\mathbb{Q}_{M}^{*}\right)$.

The terms in (53) which correspond to $L^{n} W_{\alpha}$ can now be rewritten as follows: 


$$
\begin{aligned}
\sum_{\alpha} \sum_{n=0}^{\infty} \frac{1}{2 i} \oint_{L^{n} W_{\alpha}\left(\Theta_{M}^{*}\right)} R(z) \operatorname{Tr}\left[\operatorname{Tr}(z) L^{n} W_{\alpha} M W_{\alpha}^{-1} L^{-n}\right] d z \\
\quad=\pi c_{1} \sum_{\alpha} \sum_{n=0}^{\infty} \varepsilon_{\alpha} \operatorname{Tr}\left[\Re\left(\xi_{L}\right) L^{n} W_{\alpha} M W_{\alpha}^{-1} L^{-n}\right] \quad\left(\varepsilon_{\alpha}= \pm 1\right) .
\end{aligned}
$$

Using equation (28), we easily see that:

$$
\Re\left(\xi_{L}\right)=\left(\lambda_{L}\right)^{n} \cdot L^{n} \Re\left(\xi_{L}\right) L^{-n} \text { for } n \in \mathbf{Z},
$$

where $0<\lambda_{L}<1$ is the multiplier of $L$. Consequently,

$$
\sum_{n=0}^{\infty} \operatorname{Tr}\left[\pi\left(\xi_{L}\right) L^{n} W_{\alpha} M W_{\alpha}^{-1} L^{-n}\right]=\frac{\operatorname{Tr}\left[\Re\left(\xi_{L}\right) W_{\alpha} M W_{\alpha}^{-1}\right]}{1-\lambda_{L}} .
$$

Putting everything together, we finally obtain:

$$
\operatorname{Im}(J)=[\text { a finite number of elementary terms }]
$$

$$
+\sum_{j=1}^{N} \pi c_{j}\left\{\sum_{\alpha} \varepsilon_{\alpha} \frac{\operatorname{Tr}\left[\Re\left(\xi_{j}\right) W_{\alpha} M W_{\alpha}^{-1}\right]}{1-\lambda_{L}}\right\} .
$$

Note: for simplicity, the subscript $j$ has been omitted in $\alpha, \varepsilon_{\alpha}, W_{\alpha}, \lambda_{L}$.

The first assertion in Theorem 4 is now rather obvious.

Before turning to the second assertion, one very important point should be noted. It is well known that Teichmüller space $T_{g}$ can be realized as a space of marked Fuchsian groups (20); cf. [17, pp. 14-19]. In addition, recall that the elements of $\Gamma$ can be represented as words in a formal group [corresponding to (20)]. By considering appropriate quasi-conformal mappings on $H$, we deduce that: (i) the relative position of hyperbolic fixpoints is unchanged when $\tau$ varies in $T_{g}$ [1, p. 47]; (ii) the form of $\operatorname{Im}(J)$ is independent of $\tau \in T_{g}$. The rational expressions $X_{j M}$ can therefore be determined by studying a special choice of $\Gamma$.

To show that this determination can be carried out effectively, we use the so-called Nielsen ordering on $\hat{\mathbf{R}}$ [44, pp. 193-231]. This ordering (which deserves to be much better known) is based on the fact that there is a 1-1 correspondence between points $x \in \hat{\mathbf{R}}$ and certain infinite words $\prod_{k=1}^{\infty} L_{k}$ in the group (20). In particular,

$$
x=\lim _{n \rightarrow \infty} L_{1} L_{2} \cdots L_{n}\left(z_{0}\right) \quad\left[\text { for any } z_{0} \in H\right] .
$$

Hyperbolic fixpoints correspond to words which are eventually periodic. See also [8], [16], [35], [39].

Among other things, the Nielsen ordering can be used to set up an effective procedure for determining the relative position of fixpoints. In view of this, we can easily decide which loops $W\left(\mathbb{Q}_{M}^{*}\right)$ go around which fixpoints $\xi_{j}$. These considerations ultimately lead to a rigorous proof of the second assertion.

Corollary $(n=2)$. Suppose that $R$ satisfies the hypotheses of Theorem 4. Then: $\theta[z, R] \equiv 0$ on $D$ if and only if 


$$
\sum_{j=1}^{N} c_{j} X_{j M_{k}}=0 \text { for } 1 \leqslant k \leqslant 6 g-6 .
$$

Needless to say, the elements $M_{k}$ correspond to the Fricke paths .

Proof. We merely combine Theorems 3 and 4.

Before formulating any concrete examples, it is useful to look at Theorem 3 in connection with another kind of Poincaré series.

To define these series, we first choose any primitive element $T=\left(\begin{array}{ll}a & b \\ c & d\end{array}\right)$ in $\Gamma$. We then write:

$$
Q_{T}(z)=\frac{1}{c^{2}} \sum_{W \in[T] \backslash \Gamma} \frac{W^{\prime}(z)^{2}}{\left(W z-\xi_{T}\right)^{2}\left(W z-\eta_{T}\right)^{2}}
$$

[assuming that $c \neq 0$ ].

The fixpoints of $T$ are denoted (as usual) by $\xi_{T}$ and $\eta_{T}$; the elements $W$ are understood to be coset representatives: $\Gamma=\Sigma[T] W$. For obvious reasons, $Q_{T}(z)$ is called a relative Poincaré series. The modifications required when $c=0$ are not important; they will therefore be omitted.

It is easily checked that:

(i) $\left(z-\xi_{T}\right)^{-2}\left(z-\eta_{T}\right)^{-2} \in \mathbb{Q}_{2}\left(D, \Gamma_{0}\right)$ where $\Gamma_{0}=[T]$;

(ii) $Q_{T}(z)$ is unaffected by ambiguities in the choice of $W$;

(iii) $Q_{T}(z)$ is absolutely convergent on $D$;

(iv) $Q_{T}(z) \in \mathbb{Q}_{2}(D, \Gamma)$;

(v) $Q_{T}(z)$ can be expressed as a sum over the conjugacy class $\{T\}$, viz.

$$
Q_{T}(z)=\sum_{R \in\{T\}} \frac{R^{\prime}(z)}{(z-R z)^{2}} .
$$

In view of [19], definition (56) seems rather natural. The series $Q_{T}(z)$ can also be found in [46], [47], but they appear in a disguised form. It is interesting to note that Poincare considered relative series of a somewhat different type in [55], [57].

There is a simple transformation formula for the period (26) when $M$ is replaced by $\boldsymbol{M}^{k}$. Namely:

$$
I\left[q, M^{k}\right]=k \frac{m^{k}-m^{-k}}{m-m^{-1}} I[q, M],
$$

where $m$ and $m^{-1}$ are the eigenvalues of $M \in S L(2, \mathbf{R})$. For this reason, there is usually no loss of generality if one assumes that $M$ is primitive.

THEOREM 5. Suppose that $T$ and $M$ are primitive elements in $\Gamma$ such that $\{T\} \neq\{M\}$ and $\{T\} \neq\left\{M^{-1}\right\}$. Let $\gamma_{T}$ and $\gamma_{M}$ be any curves in the free homotopy classes corresponding to $\{T\}$ and $\{M\}$. Then:

$$
\operatorname{Im} I\left[Q_{T}, M\right]=\frac{\pi \operatorname{sgn} \operatorname{Tr}(T)}{\left[\operatorname{Tr}^{2}(T)-4\right]^{3 / 2}} \sum_{k} \varepsilon_{k} \mathscr{P}\left[T, W_{k} M W_{k}^{-1}\right],
$$

where 
(a) the number of terms in the $k$-sum is $\leqslant \operatorname{card}\left(\gamma_{M} \cap \gamma_{T}\right)$;

(b) the $W_{k}$ are double coset representatives in $[T] \backslash \Gamma /[M]$ and $\varepsilon_{k}= \pm 1$;

(c) $\mathcal{P}[X, Y]=\operatorname{Tr}(X) \operatorname{Tr}(Y)-2 \operatorname{Tr}(X Y)$.

The entries $\varepsilon_{k}$ and $W_{k}$ can be determined by means of an algorithm once $\gamma_{T}$ and $\gamma_{M}$ are visualized on $F$.

Finally, if $\{T\}=\{M\}$ or $\{T\}=\left\{M^{-1}\right\}$, then $\operatorname{Im} I\left[Q_{T}, M\right]=0$.

Proof (sketch). Suppose that $\{T\} \neq\left\{M^{ \pm 1}\right\}$. The coset representatives $W \in[T] \backslash \Gamma$ can now be expressed in terms of the double coset representatives $W_{0} \in[T] \backslash \Gamma /[M]$; that is, $W=W_{0} M^{k}(k \in \mathbf{Z})$. By reviewing the proof of equation (50), we immediately see that:

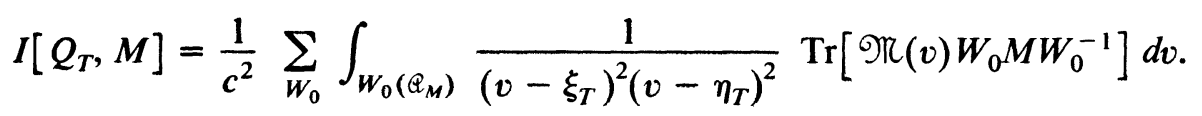

Note that the points $\xi_{T}, \eta_{T}, W_{0}\left(\xi_{M}\right), W_{0}\left(\eta_{M}\right)$ are distinct. For this reason, the integrals over $W_{0}\left(Q_{M}\right)$ are perfectly harmless. Using equation (52), we obtain:

(59) $\operatorname{Im} I\left[Q_{T}, M\right]=\frac{1}{c^{2}} \sum_{W_{0}} \frac{1}{2 i} \oint_{W_{0}\left(Q_{M}^{*}\right)} \frac{\operatorname{Tr}\left[\Re(v) W_{0} M W_{0}^{-1}\right]}{\left(v-\xi_{T}\right)^{2}\left(v-\eta_{T}\right)^{2}} d v$.

But, the $W_{0}\left(\mathscr{Q}_{M}^{*}\right)$ integrals are zero unless $W_{0}\left(\mathbb{Q}_{M}^{*}\right)$ separates $\xi_{T}$ and $\eta_{T}$. Cf. Figure 3.

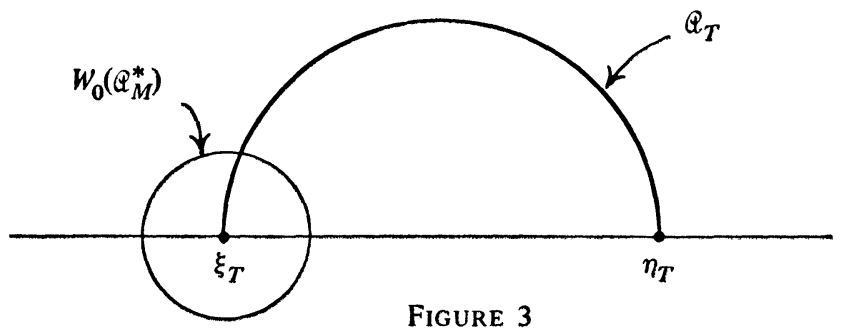

To analyze which elements $W_{0}$ are now relevant, we pass back to $F$ using $\varphi$. By deforming $\mathbb{Q}_{T}$ and $\mathbb{Q}_{M}$, we can ensure that $\gamma_{T}=\varphi\left(\mathbb{Q}_{T}\right)$ and $\gamma_{M}=\varphi\left(\mathbb{Q}_{M}\right)$. Let $\mathcal{C}_{T}$ be any subinterval along $\mathbb{Q}_{T}$ which is homeomorphic to $\gamma_{T}$ under $\varphi$. Since we can always replace $W_{0}$ by $T^{k} W_{0}$, there is no harm in assuming that $W_{0}\left(\mathbb{Q}_{M}^{*}\right)$ crosses $\mathbb{Q}_{T}$ along $\mathcal{C}_{T}$. Assertion (a) follows immediately. It is understood here that $\gamma_{T}$ and $\gamma_{M}$ intersect transversally.

Recall that $T=\left(\begin{array}{ll}a & b \\ c & d\end{array}\right)$. By means of a straightforward computation, we see that:

$$
\frac{1}{2 i} \oint_{\left|z-\xi_{T}\right|=\delta} \frac{\operatorname{Tr}[\Re(z) Q]}{\left(z-\xi_{T}\right)^{2}\left(z-\eta_{T}\right)^{2}} d z \quad \text { (counterclockwise) }
$$

$$
=c^{2} \cdot \frac{\pi \operatorname{sgn} \operatorname{Tr}(T)}{\left[\operatorname{Tr}^{2}(T)-4\right]^{3 / 2}}[2 \operatorname{Tr}(Q T)-\operatorname{Tr}(Q) \operatorname{Tr}(T)]
$$

for any $Q \in S L(2, \mathrm{C})$. The desired formula for $\operatorname{Im} I\left[Q_{T}, M\right]$ is now a trivial consequence of (59) and (60). 
Once $\gamma_{T}$ and $\gamma_{M}$ are drawn on $F$, the Nielsen ordering can be used to formulate an algorithm for determining $W_{k}$ and $\varepsilon_{k}$. Cf. the last three paragraphs in the proof of Theorem 4 . This algorithm is particularly simple when $\gamma_{T}$ and $\gamma_{M}$ are Jordan curves.

The exceptional cases $\{T\}=\left\{M^{ \pm 1}\right\}$ can be treated by means of a special [but similar] argument.

Theorem 5 can be used to construct explicit bases for $\mathbb{Q}_{2}(H, \Gamma)_{\mathbf{R}}$. To do so, one merely guesses $6 g-6$ relative Poincare series $Q_{T_{j}}(z)$ and then tries to verify (by explicit computation) that:

$$
\operatorname{rank}\left[\operatorname{Im} I\left[Q_{T_{j}}, M_{k}\right]\right]=6 g-6 .
$$

Note that this equation kills two birds with one stone: (a) $\left\{Q_{T_{j}}\right\}_{j=1}^{6 g-6}$ will be a basis for $\mathbb{C}_{2}(H, \Gamma)_{\mathbf{R}}$; (b) Theorem 3 will be proved by direct computation. For generic $\Gamma$ and $2<g \leqslant 5$, we easily see that $T_{j}=M_{j}$ works. The same result presumably holds for all $g \geqslant 6$. [The proof reduces to showing that a certain $(4 g-6) \times(4 g-6)$ determinant defined over $S L(2, C)^{2 g-2} \times C^{2}$ is not identically zero.]

Once (61) is established, we can set

$$
Q_{T}(z)=\sum_{j=1}^{6 g-6} c_{j} Q_{T_{j}}(z) \quad\left[c_{j} \in \mathbf{R}\right]
$$

and then solve for the $c_{j}$ by means of linear algebra in the field $\mathbf{Q}\left(\operatorname{tr} \Gamma, \sqrt{\operatorname{tr}^{2}(\Gamma)-4}\right)$. To prove this, we simply observe that:

$$
\operatorname{Im} I\left[Q_{T}, M_{k}\right]=\sum_{j=1}^{6 g-6} c_{j} \operatorname{Im} I\left[Q_{T_{j}}, M_{k}\right], \quad 1 \leqslant k \leqslant 6 g-6 .
$$

Compare [19, Theorem 5]. To get some feeling for the $c_{j}$ in (62)-(63), we include the following period table for $g=2$.

\begin{tabular}{l|cccccc|} 
& \multicolumn{1}{c}{$A_{1}$} & $B_{1}$ & $A_{2}$ & $B_{2}$ & $\left(A_{1}, B_{1}\right)$ & $A_{1} A_{2}$ \\
\hline$Q_{A_{1}}$ & 0 & $P\left[A_{1}, B_{1}\right]$ & 0 & 0 & 0 & 0 \\
$Q_{B_{1}}$ & $-P\left[A_{1}, B_{1}\right]$ & 0 & 0 & 0 & 0 & $P\left[A_{2} A_{1}, B_{1}\right]$ \\
$Q_{A_{2}}$ & 0 & 0 & 0 & $P\left[A_{2}, B_{2}\right]$ & 0 & 0 \\
$Q_{B_{2}}$ & 0 & 0 & $-P\left[A_{2}, B_{2}\right]$ & 0 & 0 & $P\left[A_{1} A_{2}, B_{2}\right]$ \\
$Q_{\left.1 A_{1}, B_{1}\right)}$ & 0 & 0 & 0 & 0 & 0 & $\left.2 D\left[A_{1}, B_{1}\right), A_{1}, A_{2}\right]$ \\
$Q_{A_{1}, A_{2}}$ & 0 & $-P\left[A_{2} A_{1}, B_{1}\right]$ & 0 & $-P\left[A_{1} A_{2}, B_{2}\right]$ & $-2 D\left[\left(A_{1}, B_{1}\right), A_{1}, A_{2}\right]$ & 0 \\
\hline$Q_{T}$ & \multicolumn{7}{c}{} & & & \\
\hline
\end{tabular}

The following notational conventions hold: (a) $\mathcal{P}[X, Y]=\operatorname{Tr}(X) \operatorname{Tr}(Y)-$ $2 \operatorname{Tr}(X Y)$; (b) $D[U, V, W]=\operatorname{Tr}(U V W)-\operatorname{Tr}(U W V)$; (c) the factor $\pi\left[\operatorname{Tr}^{2}(T)\right.$ $-4]^{-3 / 2}$ sgn $\operatorname{Tr}(T)$ is omitted in each row.

Remark [about the Fuchsian parameters]. Assume, for a moment, that the $c_{j}$ in equation (62) are given by reasonably simple formulas. It is now very tempting to formulate a Fuchsian analog of $[19, \$ 4]$. In the present case, the Fuchsian parameters $f_{j}$ must satisfy:

$$
3 l(z, z)=\sum_{j=1}^{6 g-6} f_{j} R_{j}(z) \quad \text { for } z \in H,
$$


where $Q_{j} d x^{2}=R_{j}(z) d z^{2}$ as in equations (11) and (26). A purely formal computation starting with

$$
\begin{aligned}
\Pi_{\xi \eta}^{2 w} & =\sum_{T \in \Gamma} \log \frac{(T z-\xi)(T w-\eta)}{(T z-\eta)(T w-\xi)} \\
& =[\text { an Abelian integral of the third kind }]
\end{aligned}
$$

yields

$$
l(z, \xi)=\sum_{T \in \Gamma-\{I\}} \frac{T^{\prime}(z)}{[T z-\xi]^{2}} .
$$

Unfortunately, this formal expression for $l(z, \xi)$ is not absolutely convergent. [Proof: integrate over $\mathcal{F}$ with respect to $y^{-1} d x d y$; note that there is a logarithmic divergence at $y=0$.] Nevertheless, it seems entirely possible that the expansion for $l(z, \xi)$ will be conditionally convergent (at least when its terms are added in a special order). Compare: [24, p. 154], [42], [43], [49], [50], [67]. By modifying the arguments in [19, §4], we immediately obtain explicit formulas for the $f_{j}$ in terms of $\Gamma$ and $\operatorname{Im} I\left[R_{j}, M_{k}\right]$. These formulas involve some messy infinite series which appear to be conditionally convergent at best. [The case of bordered Riemann surfaces may be easier; see Problem (III) at the end of §7.]

Further investigation along these lines is definitely called for. The crucial point seems to be that the $f_{j}$ should be regarded as functions of $\Gamma$, not $F$. We recall that a similar trick is used in the theory of elliptic functions; i.e. Jacobi inversion. Cf. [15, p. 174].

Finally, there is an interesting connection with the Petersson inner product which should be mentioned. Suppose that $M \in \Gamma$ is primitive and that $f \in \mathbb{Q}_{2}(H, \Gamma)$. Then:

$$
\left(f, Q_{M}\right)=-\frac{1}{2} \pi \frac{\operatorname{sgn} \operatorname{Tr}(M)}{\left[\operatorname{Tr}^{2}(M)-4\right]^{3 / 2}} \int_{z_{0}}^{M z_{0}} f(z) \operatorname{Tr}[\pi(z) M] d z
$$

Compare: equations (43), (77), and [47, pp. 37-41].

We can now formulate several concrete examples. For convenience, it will be assumed that certain hyperbolic fixpoints are finite. Conditions of this type can always be fulfilled by making a suitable conjugation. Cf. equations (22)-(23).

Example 1 (an illustration of Theorem 4). Consider the case $g=2$, $\Gamma=\left[A_{1}, A_{2} ; B_{1}, B_{2}\right]$. Set:

$$
R(z)=\frac{\left(b-b_{1}\right)\left(b-b_{2}\right)\left(b-b_{3}\right)}{(z-b)\left(z-b_{1}\right)\left(z-b_{2}\right)\left(z-b_{3}\right)},
$$

where $\left(b, b_{1}, b_{2}, b_{3}\right)=\left(\xi_{B_{1}}, \xi_{A_{1}}, \eta_{B_{1}}, \eta_{A_{1}}\right) \in \mathbf{R}^{4}$. In addition, write:

$$
R(z)=\frac{c\left(B_{1}^{+}\right)}{z-\xi_{B_{1}}}+\frac{c\left(A_{1}^{+}\right)}{z-\xi_{A_{1}}}+\frac{c\left(B_{1}^{-}\right)}{z-\eta_{B_{1}}}+\frac{c\left(A_{1}^{-}\right)}{z-\eta_{A_{1}}} .
$$


Using Theorem 4 and the Nielsen ordering, we can compute the following period table.

\begin{tabular}{|l|l|}
\hline$M_{k}$ & $\left.\frac{1}{\pi} \operatorname{Im} \operatorname{I} \theta(z, R), M_{k}\right]$ \\
\hline$A_{1}$ & $c\left(B_{1}^{+}\right) \frac{\lambda_{B_{1}}}{1-\lambda_{B_{1}}} \operatorname{Tr}\left[\pi\left(\xi_{B_{1}}\right) A_{1}\right]-c\left(B_{1}^{-}\right) \frac{1}{1-\lambda_{B_{1}}} \operatorname{Tr}\left[\Re\left(\eta_{B_{1}}\right) A_{1}\right]$ \\
$B_{1}$ & $c\left(A_{1}^{-}\right) \frac{\lambda_{A_{1}}}{1-\lambda_{A_{1}}} \operatorname{Tr}\left[\Re\left(\eta_{A_{1}}\right) B_{1}\right]-c\left(A_{1}^{+}\right) \frac{1}{1-\lambda_{A_{1}}} \operatorname{Tr}\left[\Re\left(\xi_{A_{1}}\right) B_{1}\right]$ \\
$A_{2}$ & 0 \\
$B_{2}$ & 0 \\
$\left(A_{1}, B_{1}\right)$ & 0 \\
$A_{1} A_{2}$ & $c\left(B_{1}^{+}\right) \frac{1}{1-\lambda_{B_{1}}} \operatorname{Tr}\left[\Re\left(\xi_{B_{1}}\right) A_{2} A_{1}\right]-c\left(B_{1}^{-}\right) \frac{\lambda_{B_{1}}}{1-\lambda_{B_{1}}} \operatorname{Tr}\left[\Re\left(\eta_{B_{1}}\right) A_{2} A_{1}\right]$ \\
\hline
\end{tabular}

[Recall that $0<\lambda_{L}<1$ is the multiplier of $L$.] As shown in [18, Theorem 7.14], the elements $M_{k}$ correspond to legitimate Fricke paths whenever

$$
\left\{\begin{array}{c}
\operatorname{Tr}\left(A_{1}\right) \operatorname{Tr}\left(B_{1}\right) \neq 2 \operatorname{Tr}\left(A_{1} B_{1}\right), \quad \operatorname{Tr}\left(A_{2}\right) \operatorname{Tr}\left(B_{2}\right) \neq 2 \operatorname{Tr}\left(A_{2} B_{2}\right) \\
D\left[\left(A_{1}, B_{1}\right), A_{1}, A_{2}\right] \neq 0
\end{array}\right\} .
$$

Compare: (24) and (64).

EXAMPLE 2. The completeness theorem of Bers [mentioned after (44)] can easily be strengthened so as to provide an explicit set $\left\{\theta\left[z, R_{j}\right]: 1 \leqslant j \leqslant N\right\}$ which spans $\mathbb{Q}_{n}(D, \Gamma)_{\mathbf{C}}$. In fact, using the notation of [34, p. 383], it is enough to look at $R_{j}(z)=\varphi(u ; z)$ with $u \in\left\{A_{k}\left(a_{\sigma}\right), B_{k}\left(a_{\sigma}\right)\right\}$ and $1 \leqslant k \leqslant g, 1 \leqslant \sigma$ $\leqslant 2 n-1$. Accordingly, the number $N$ will not exceed $2 g(2 n-1)$.

We consider the case $n=2, g=2, \Gamma=\left[A_{1}, A_{2} ; B_{1}, B_{2}\right]$ and suppose that (66) holds. Set:

$$
\begin{aligned}
R_{j}(z)=\frac{\left(c_{j}-b_{1}\right)\left(c_{j}-b_{2}\right)\left(c_{j}-b_{3}\right)}{\left(z-c_{j}\right)\left(z-b_{1}\right)\left(z-b_{2}\right)\left(z-b_{3}\right)} \\
\text { where }\left(b_{1}, b_{2}, b_{3}\right)=\left(\xi_{A_{1}}, \eta_{B_{1}}, \eta_{A_{1}}\right) \in \mathbf{R}^{3} .
\end{aligned}
$$

To apply the extended version of Bers' theorem, we define:

$$
\left\{\begin{array}{ccc}
* & c_{1}=A_{1}\left(\eta_{B_{1}}\right) & * \\
c_{2}=A_{2}\left(\xi_{A_{1}}\right) & c_{3}=A_{2}\left(\eta_{B_{1}}\right) & c_{4}=A_{2}\left(\eta_{A_{1}}\right) \\
c_{5}=B_{1}\left(\xi_{A_{1}}\right) & * & c_{6}=B_{1}\left(\eta_{A_{1}}\right) \\
c_{7}=B_{2}\left(\xi_{A_{1}}\right) & c_{8}=B_{2}\left(\eta_{B_{1}}\right) & c_{9}=B_{2}\left(\eta_{A_{1}}\right)
\end{array}\right\} .
$$


It is understood here that $c_{j} \neq \infty$. [The missing entries correspond to trivia.] Using Theorem 4 and the Nielsen ordering, we can now compute Im $I\left[\theta\left(z, R_{j}\right), M_{k}\right]$ as in Example 1. An explicit (but rather lengthy) computation will ultimately show that:

$\operatorname{rank}\left[\operatorname{Im} I\left[\theta\left(z, R_{j}\right), M_{k}\right]\right]=6$ for $j \in\{1,2,4,5,6,7\}$ and $1<k<6$, provided

$$
\begin{gathered}
D\left[A_{1}, A_{2} A_{1}, B_{1} A_{1} B_{1}^{-1}\right] \neq 0 \text { and } \\
D\left[B_{2},\left(A_{1}, B_{1}\right), A_{1}\right] \neq D\left[B_{2}, A_{2}^{-1}\left(A_{1}, B_{1}\right) A_{2}, A_{1}\right] .
\end{gathered}
$$

It is easily seen that conditions (66)-(67) define a dense subset of Teichmüller space. Subject to these restrictions, we conclude that $\left\{\theta\left[z, R_{j}\right]: j=\right.$ $1,2,4,5,6,7\}$ is a basis for both $\mathbb{Q}_{2}(H, \Gamma)_{\mathbf{R}}$ and $\mathbb{Q}_{2}(D, \Gamma)_{\mathbf{C}}$. Cf. equations (48)-(49).

Taking things a bit further, we now write:

$$
\theta\left[z, R_{3}\right]=\sum_{j} \lambda_{j} \theta\left[z, R_{j}\right] \text { with } j \in\{1,2,4,5,6,7\} \text { and } \lambda_{j} \in \mathbf{R} \text {. }
$$

To solve for $\lambda_{j}$, it is sufficient to look at the $M_{k}$ periods:

$$
\operatorname{Im} I\left[\theta\left(z, R_{3}\right), M_{k}\right]=\sum_{j} \lambda_{j} \operatorname{Im} I\left[\theta\left(z, R_{j}\right), M_{k}\right], \quad 1<k<6 .
$$

In particular, by taking $k=3$, we find that $\lambda_{7}=0$. Hence:

$$
\begin{aligned}
\theta\left[z, R_{3}\right]= & \lambda_{1} \theta\left[z, R_{1}\right]+\lambda_{2} \theta\left[z, R_{2}\right]+\lambda_{4} \theta\left[z, R_{4}\right] \\
& +\lambda_{5} \theta\left[z, R_{5}\right]+\lambda_{6} \theta\left[z, R_{6}\right] .
\end{aligned}
$$

This curious identity shows that $\left\{\theta\left[z, R_{j}\right]\right\}_{j=1}^{6}$ is not a basis for $\mathcal{Q}_{2}(D, \Gamma)$.

Conclusion: some caution is required whenever we try to extract a basis from the explicit set $\left\{\theta\left[z, R_{j}\right]: 1<j<N\right\}$. Compare [48].

EXAMPLe 3 (a REMARKABle IDENTITY). Assume once again that $\mathbf{g}=\mathbf{2}$, $\Gamma=\left[A_{1}, A_{2} ; B_{1}, B_{2}\right]$. Furthermore, suppose that (66) holds. Then, in view of period table (64), $\left\{Q_{M_{k}}(z)\right\}_{k=1}^{6}$ will be an explicit basis for $Q_{2}(H, \Gamma)_{R}$.

Let $R_{j}(z)$ be defined as in Example 2. Since the periods $\operatorname{Im} I\left[\theta\left(z, R_{j}\right), M_{k}\right]$ can be computed explicitly, it is trivial to express $\theta\left[z, R_{j}\right]$ in terms of $\left\{Q_{M_{k}}\right\}_{k=1}^{6}$. Taking $j=1$, we obtain:

$$
\left\{\begin{array}{c}
\theta\left[z, R_{1}\right]=c Q_{A_{1}}(z) \\
c=\left[\operatorname{Tr}^{2}\left(A_{1}\right)-4\right] \cdot \frac{\left(A_{1} \eta_{B_{1}}-\xi_{A_{1}}\right)\left(A_{1} \eta_{B_{1}}-\eta_{A_{1}}\right)}{\xi_{A_{1}}-\eta_{A_{1}}}
\end{array}\right\} .
$$

To eliminate the restriction (66), we simply apply a continuity argument.

7. Results for $n$th order differentials [ $n>2$ ]. In this section, we shall be content to give a leisurely outline of the main results. Since brute-force computations are needed in the derivation of several formulas, it will be convenient to assume that: 


$$
n<6 \text {. }
$$

This restriction can almost certainly be removed; cf. the paragraph following Theorem 11.

The basic idea is now rather obvious. We consider $\varphi \in \mathbb{Q}_{n}(H, \Gamma)_{\mathbb{R}}$ and then try to develop a period $I_{n}[\varphi, M]$ which generalizes (26). This period should be computable in the sense that there are analogs of Theorems 4 and 5. But, more importantly, there should also be an analog of Theorem 3. The extension of Theorem 3 causes serious problems because it is not at all clear what the generalized Fricke paths $\left\{M_{k}\right\}_{k=1}^{(2 n-1)(2 g-2)}$ will be [cf. equations (22) $-(24)$ and (78)-(80)]. We will say more about this matter in a few minutes.

To discover the correct formula for $I_{n}[\varphi, M]$, we need a little background material. Cf. [20]. We fix $n \geqslant 2$ and then define the column vectors

$$
X(z)=\left(z^{n-1-\alpha}\right), \quad Y(z)=\left((-1)^{\alpha}\left(\begin{array}{c}
n-1 \\
\alpha
\end{array}\right) z^{\alpha}\right),
$$

where $0<a<n-1$. We also define

$$
\mathscr{R}(z)=X(z) Y(z)^{t} \quad[t=\text { transpose }] .
$$

Note: when $n=2$, this $\Re$ differs from (27) by a factor of $(-1)$.

For any matrix $T=\left(\begin{array}{cc}a & b \\ c & d\end{array}\right) \in S L(2, \mathrm{C})$, observe that there is a uniquely determined $n \times n$ matrix $\chi(T)$ such that:

$$
X(T z)(c z+d)^{n-1}=\chi(T) X(z) .
$$

We set $\mathcal{Y}(T)=\chi\left(T^{-1}\right)^{t}$.

FACr. The following properties hold:

(i) $X(T z)(c z+d)^{n-1}=\chi(T) X(z)$ and $Y(T z)(c z+d)^{n-1}=\mathcal{Y}(T) Y(z)$;

(ii) $\chi$ and $\mathcal{Y}$ are homomorphisms $S L(2, \mathrm{C}) \rightarrow S L(n, \mathrm{C})$;

(iii) $\Re(T z) T^{\prime}(z)^{1-n}=\chi(T) \Re(z) \chi\left(T^{-1}\right)$;

(iv) $\operatorname{Tr}[\operatorname{TR}(z) \chi(T)]=(-1)^{n-1}\left[c z^{2}+(d-a) z-b\right]^{n-1}$;

(v) $\mathscr{T}(z)=\left[\mathfrak{T}_{\alpha \beta}(z)\right]$ where $\mathfrak{T}_{\alpha \beta}(z)=(-1)^{\beta}\left(\begin{array}{c}n-1 \\ \beta\end{array}\right) z^{n-1+\beta-\alpha}$ and $0<$ $\alpha, \beta<n-1$.

As explained in [18], [21], we can now generalize (26) by writing:

$$
I_{n}[\varphi, M]=-\int_{z_{0}}^{M z_{0}} \varphi(z) \operatorname{Tr}[\operatorname{TR}(z) \chi(M)] d z
$$

$$
\text { for } M \in \Gamma \text { and } \varphi \in \mathbb{Q}_{n}(H, \Gamma) \text {. }
$$

In view of assertion (iii), there is no problem in verifying the analog of (29). Furthermore, since the geometry is independent of $n$, there will be immediate analogs of Theorems 4 and 5 once we define

$$
Q_{T}(z)=\frac{1}{c^{n}} \sum_{W \in[T] \backslash \Gamma} \frac{W^{\prime}(z)^{n}}{\left(W z-\xi_{T}\right)^{n}\left(W z-\eta_{T}\right)^{n}} .
$$

Cf. [46], [47] and note that statements (i)-(v) following equation (56) carry over with trivial modifications. In particular, 


$$
Q_{T}(z)=\sum_{R \in\{T\}} \frac{R^{\prime}(z)^{n / 2}}{(z-R z)^{n}} .
$$

The analogs of (60) and (65) turn out to be:

$$
\left\{\begin{array}{c}
\frac{1}{2 i} \oint_{\left|z-\xi_{T}\right|=\delta} \frac{\operatorname{Tr}[\mathscr{T}(z) \chi(Q)]}{\left(z-\xi_{T}\right)^{n}\left(z-\eta_{T}\right)^{n}} d z=c^{n} \cdot \frac{\pi \operatorname{sgn} \operatorname{Tr}(T)}{\left[\operatorname{Tr}^{2}(T)-4\right]^{n-1 / 2}} \mathscr{P}[Q, T] \\
\mathscr{P}[X, Y]=\sum_{2 r+s=n-1} \frac{(n-1) !}{r ! s ! r !}[\operatorname{Tr}(X) \operatorname{Tr}(Y)-2 \operatorname{Tr}(X Y)]^{s}[\operatorname{Tr}(X, Y)-2]^{r}
\end{array}\right\}
$$

and

$$
\left(f, Q_{M}\right)=e^{\pi i(n-1)} \frac{\Gamma\left(\frac{1}{2}\right) \Gamma\left(n-\frac{1}{2}\right)}{\Gamma(n)} \frac{\operatorname{sgn} \operatorname{Tr}(M)}{\left[\operatorname{Tr}^{2}(M)-4\right]^{n-1 / 2}} I_{n}[f, M]
$$

$$
\text { for } f \in \mathbb{Q}_{n}(H, \Gamma) \text {. }
$$

To complete the picture, it remains to generalize Theorem 3 . This is where the trouble begins!

Loosely speaking, there are two possibilities:

(A) the computational approach, where we try to generalize equation (61) by means of educated guessing and explicit computation;

(B) the theoretical approach, where we try to generalize Theorem 3 [without any guesswork] by first determining the geometric significance of the generalized Fricke paths $\boldsymbol{M}_{\boldsymbol{k}}$.

REMARK. In method (B), no attempt is made to find an explicit basis for $\mathbb{Q}_{n}(H, \Gamma)_{\mathrm{R}}$.

Before stating any theorems, it may be useful to take a quick look at [some of ] the difficulties encountered in both methods.

First of all, notice that two educated guesses are required in method (A). That is, one must choose $(2 n-1)(2 g-2)$ relative Poincaré series $Q_{T_{j}}(z)$ and an equal number of matrices $M_{k} \in \Gamma$. There is nothing to dictate these choices when $n \geqslant 3$. As a result, the rank of our new period matrix can fail to be maximal for at least two reasons: (i) when the $Q_{T_{j}}$ do not span $\mathbb{Q}_{n}(H, \Gamma)_{\mathrm{R}}$; and (ii) when the elements $\boldsymbol{M}_{k}$ are "interrelated" in some sense [e.g. conjugate].

For small values of $g$ and $n$, it is very tempting to make some informal choices of $Q_{T_{j}}$ and $M_{k}$, and then try to compute the desired rank numerically [at least for generic $\Gamma$ ]. Although this project is entirely feasible for $(g, n)=$ $(2,3)$ and $(2,4)$, the penalty for bad guesses is very high. I learned this the hard way. After three weeks of meticulous computation, my first nine experiments all managed to end in failure! As I discovered later, the difficulties were caused solely by making bad choics of $\boldsymbol{M}_{\boldsymbol{k}}$. [You might say my early guesses were not educated enough.]

Conclusion (A). For this numerical method to be practical, one must find some way to narrow down the choice of $\left\{M_{k}\right\}_{\mathrm{k}=1}^{(2 n-1)(2 g-2)}$. 
Turning to method (B), notice that the fact preceding equation (24) supplies a geometric interpretation for the $M_{k}$ when $n=2$. It would be nice if we could prove a similar result for $n \geqslant 3$. With this objective in mind, we define:

$$
\begin{aligned}
& \text { (78) } N(g, n)=\left\{\left[X_{\alpha} ; Y_{\beta}\right] \in S L(n, \mathbf{C})^{2 g}:\left(X_{1}, Y_{1}\right) \cdots\left(X_{g}, Y_{g}\right)=I\right\} ; \\
& \text { (79) } \frac{N(g, n)}{S L(n, \mathbf{C})}=N(g, n)[\text { modulo inner automorphisms }] .
\end{aligned}
$$

Gunning [13], [14] has studied both of these varieties. By counting the constants, we immediately see that:

$$
\operatorname{dim}_{\mathbf{C}}\left[\frac{N(g, n)}{S L(n, \mathbf{C})}\right]=\left(n^{2}-1\right)(2 g-2) .
$$

Conclusion (B). Since $\left(n^{2}-1\right)(2 g-2)>(2 n-1)(2 g-2)$ for $n \geqslant 3$, the geometric interpretation of $\left\{\boldsymbol{M}_{k}\right\}_{k=1}^{(2 n-1)(2 g-2)}$ will have to involve more than just a trivial extension of the $\{n=2\}$ result. [One is tempted to consider subvarieties of $N(g, n) / S L(n, \mathrm{C})$.]

To discover the "real" meaning of the generalized Fricke paths, we must return to $N(g, n) / S L(n, \mathrm{C})$ and look at $n$th order linear differential equations over $H / \Gamma$. This procedure is motivated by the following coincidence:

$$
\operatorname{dim}_{\mathbf{C}}\left[\frac{N(g, n)}{S L(n, \mathbf{C})}\right]=\sum_{k=2}^{n} \operatorname{dim}_{\mathbf{R}} \mathfrak{Q}_{k}(H, \Gamma) .
$$

The sum on the right can be interpreted as the number of real accessory parameters in an $n$th order linear equation; cf. [23].

To be more precise, we recall that a linear differential equation

$$
u^{(n)}+\sum_{k=2}^{n}\left(\begin{array}{l}
n \\
k
\end{array}\right) P_{k}(z) u^{(n-k)}=0 \quad \text { [with holomorphic coefficients] }
$$

is defined over $H / \Gamma$ if and only if its solution vector $\mathscr{U}(z)$ transforms appropriately under $\Gamma$; that is,

$$
\mathcal{Q}(L z)=\hat{\chi}(L) \mathcal{Q}(z)(c z+d)^{1-n} \text { for } L=\left(\begin{array}{ll}
a & b \\
c & d
\end{array}\right) \in \Gamma \text {. }
$$

Cf. equation (32). By studying the Wronskian of $\mathscr{Q}$, we immediately see that $\hat{\chi}(L) \in S L(n, \mathbf{C})$. The mapping $L \rightarrow \hat{\chi}(L)$ is known as the monodromy homomorphism. In view of (83), the coefficients $P_{k}(z)$ must satisfy certain identities. These identities imply that there is a one-to-one correspondence between admissible $\left[P_{2}(z), \ldots, P_{n}(z)\right]$ and $\mathbb{Q}_{2}(H, \Gamma) \oplus \cdots \oplus \mathbb{Q}_{n}(H, \Gamma)$. This is where the accessory parameters enter the picture.

THEOREM 6 [for $2 \leqslant n \leqslant 6$ ]. The differential equation (82) is defined over $H / \Gamma$ if and only if 


$$
\left\{\begin{array}{c}
P_{2} \in \mathbb{Q}_{2}(H, \Gamma) \\
P_{3}-\frac{3}{2} P_{2}^{\prime} \in \mathbb{Q}_{3}(H, \Gamma) \\
P_{4}-2 P_{3}^{\prime}+\frac{6}{5} P_{2}^{\prime \prime} \in \mathbb{Q}_{4}(H, \Gamma) \\
P_{5}-\frac{5}{2} P_{4}^{\prime}+\frac{15}{7} P_{3}^{\prime \prime}-\frac{5}{7} P_{2}^{\prime \prime \prime} \in \mathbb{Q}_{5}(H, \Gamma) \\
P_{6}-3 P_{5}^{\prime}+\frac{10}{3} P_{4}^{\prime \prime}-\frac{5}{3} P_{3}^{\prime \prime \prime}+\frac{5}{14} P_{2}^{\prime \prime \prime} \in \mathbb{Q}_{6}(H, \Gamma)
\end{array}\right\} \text {. }
$$

It is understood that this list terminates after $P_{n}$.

According to Wilczynski [70, pp. 26, 32], the general formula will be:

$$
\frac{1}{2} \sum_{s=0}^{m-2}(-1)^{s} \frac{(m-2) ! m !(2 m-2-s) !}{(m-1-s) !(m-s) !(2 m-3) ! s !} P_{m-s}^{(s)} \in \mathbb{Q}_{m}(H, \Gamma) .
$$

Compare: [60, pp. 175-199]. These two references illustrate an important connection with classical invariant theory.

Motivated by equations (33)-(34), we shall now consider the variational theory of these $n$th order equations. For $2 \leqslant n \leqslant 6$, we introduce the accessory parameters $\left\{\lambda_{\alpha}, \lambda_{\beta}, \lambda_{\gamma}, \lambda_{\iota}, \lambda_{\kappa}\right\}$ by writing (as appropriate):

$$
\left\{\begin{array}{c}
P_{2}=\Sigma \lambda_{\alpha} q_{\alpha}(z) \\
P_{3}-\frac{3}{2} P_{2}^{\prime}=\Sigma \lambda_{\beta} r_{\beta}(z) \\
P_{4}-2 P_{3}^{\prime}+\frac{6}{5} P_{2}^{\prime \prime}=\Sigma \lambda_{\gamma} s_{\gamma}(z) \\
P_{5}-\frac{5}{2} P_{4}^{\prime}+\frac{15}{7} P_{3}^{\prime \prime}-\frac{5}{7} P_{2}^{\prime \prime \prime}=\Sigma \lambda_{\iota} t_{\iota}(z) \\
P_{6}-3 P_{5}^{\prime}+\frac{10}{3} P_{4}^{\prime \prime}-\frac{5}{3} P_{3}^{\prime \prime \prime}+\frac{5}{14} P_{2}^{\prime \prime \prime \prime}=\Sigma \lambda_{\kappa} w_{\kappa}(z)
\end{array}\right\} \text {. }
$$

The functions $q_{\alpha}, \ldots, w_{\kappa}$ are understood here to be basis vectors for $\mathbb{Q}_{k}(H, \Gamma)_{\mathbf{R}}, 2<k \leqslant 6$.

To normalize the solution vector $\mathcal{Q}$, we choose any point $z_{0} \in H$ and then insist that

$$
\left[\mathcal{U}\left(z_{0}\right), \mathcal{U}^{\prime}\left(z_{0}\right), \ldots, \mathcal{Q}^{(n-1)}\left(z_{0}\right)\right]=R
$$

for a fixed matrix $R \in G L(n, \mathrm{C})$. After introducing the Wronskian matrix $\Delta(z)=\left[\mathcal{U}(z), \mathcal{U}^{\prime}(z), \ldots, \mathcal{Q}^{(n-1)}(z)\right]$, we can define the adjoint vector $\mathscr{W}(z)$ by writing:

$$
\mathscr{W}(z)=\left[\begin{array}{c}
\omega_{1}(z) \\
\vdots \\
\omega_{n}(z)
\end{array}\right]=\left[n \text {th row of } \Delta(z)^{-1}\right]^{t} .
$$

The column vector $\mho(z)$ transforms according to the following rule: 
(88)

$$
\mathscr{W}(L z)=\hat{\mathcal{Y}}(L) \mathscr{W}(z)(c z+d)^{1-n} \text {, }
$$

$$
\text { for } L=\left(\begin{array}{ll}
a & b \\
c & d
\end{array}\right) \in \Gamma \text { and } \hat{\mathcal{Y}}(L)=\hat{\chi}\left(L^{-1}\right)^{t} .
$$

THEOREM 7 [for $2 \leqslant n \leqslant 6$ ]. Subject to (86), the following variational formula holds:

$$
\frac{\partial \hat{\chi}(L)}{\partial \lambda_{*}}=-\int_{z_{0}}^{L z_{0}} E_{*}(z)^{\mathscr{W}} \mho(z)^{t} d z \cdot \hat{\chi}(L)
$$

where

$$
\begin{aligned}
& E_{\alpha}(z)=q_{\alpha} \text { थ for } n=2 \text {; } \\
& \left\{\begin{array}{l}
E_{\alpha}(z)=3 q_{\alpha} \mathcal{U}^{\prime}+\frac{3}{2} q_{\alpha}^{\prime} \mathcal{U} \\
E_{\beta}(z)=r_{\beta} \mathcal{Q}
\end{array}\right\} \text { for } n=3 ; \\
& \left\{\begin{array}{l}
E_{\alpha}(z)=6 q_{\alpha} \mathcal{U}^{\prime \prime}+6 q_{\alpha}^{\prime} \mathcal{U}^{\prime}+\frac{9}{5} q_{\alpha}^{\prime \prime} \text { U } \\
E_{\beta}(z)=4 r_{\beta} \mathcal{U}^{\prime}+2 r_{\beta}^{\prime} \text { U } \\
E_{\gamma}(z)=s_{\gamma} \text { }
\end{array}\right\} \text { for } n=4 ;
\end{aligned}
$$

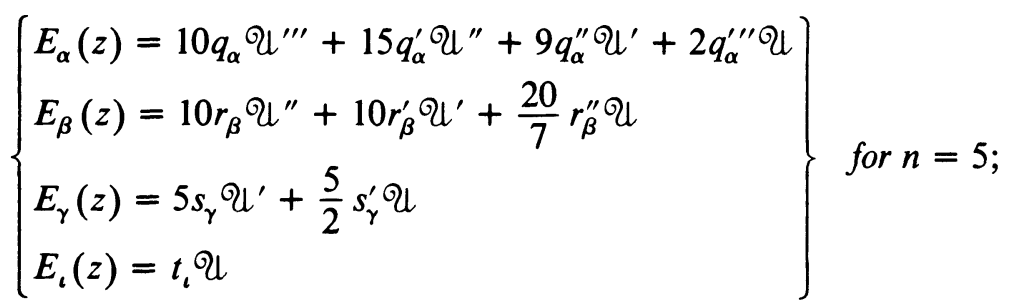

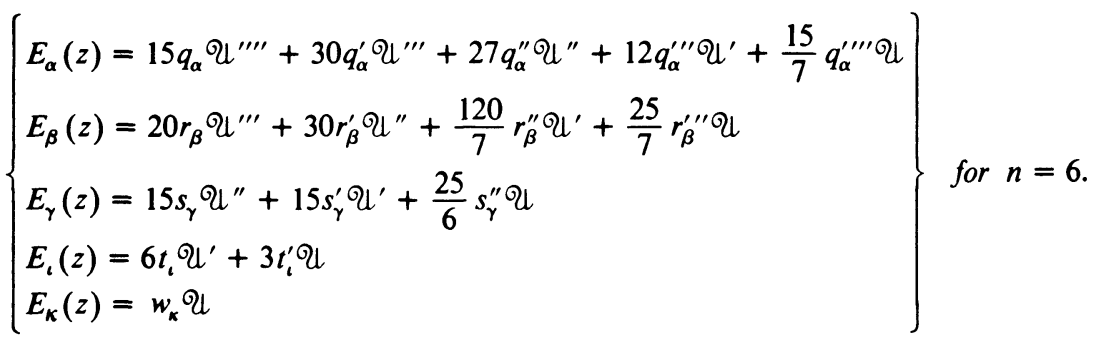

We intend to apply Theorem 7 near the trivial equation $u^{(n)}=0$. Compare: equations (30)-(31). By correctly choosing the matrix $R$ in (86), we can certainly ensure that $\mathcal{Q}(z)=X(z)$ when $u^{(n)}=0$. But,

$$
\mathscr{U}(z)=X(z) \Rightarrow \mho(z)=\frac{1}{(n-1) !} Y(z) \text {. }
$$

To obtain generalizations of the period (26), we can now mimic equation (34) and evaluate 
(90)

$$
\left\{\begin{array}{c}
I_{n}\left[q_{\alpha}, L\right] \stackrel{\text { def }}{=}(n-1) ! \frac{\partial}{\partial \lambda_{\alpha}} \operatorname{Tr} \hat{\chi}(L) \\
I_{n}\left[r_{\beta}, L\right] \stackrel{\text { def }}{=}(n-1) ! \frac{\partial}{\partial \lambda_{\beta}} \operatorname{Tr} \hat{\chi}(L) \\
\ldots
\end{array}\right\}
$$

at the trivial equation $u^{(n)}=0$. For convenience, we also define:

$$
\begin{aligned}
V_{n} & =\mathscr{C}_{2}(H, \Gamma)_{\mathbf{R}} \oplus \cdots \oplus \mathbb{Q}_{n}(H, \Gamma)_{\mathbf{R}} ; \\
N & =\left(n^{2}-1\right)(2 g-2)=\operatorname{dim}\left(V_{n}\right) .
\end{aligned}
$$

THEOREM 8 [for $2 \leqslant n \leqslant 6$ ]. These new periods $I_{n}$ are given by the formula

$$
-\int_{z_{0}}^{L z_{0}} \operatorname{Tr}\left[F_{*}(z) Y(z)^{t} \chi(L)\right] d z,
$$

where $F_{*}(z)$ is simply $E_{*}(z)$ with Q replaced by $X$ [cf. Theorem 7]. There is an immediate analog of (29) for each of these integrals. In addition, the following reduction formulas hold:

$$
\begin{aligned}
& I_{3}\left[q_{\alpha}, L\right]=3 \operatorname{Tr}(L) I_{2}\left[q_{\alpha}, L\right] \\
& \left\{\begin{array}{l}
\left.I_{4}\left[q_{\alpha}, L\right]=\frac{18}{5}\left[4+3 \operatorname{Tr}\left(L^{2}\right)\right] I_{2}\left[q_{\alpha}, L\right]\right\} ; \\
I_{4}\left[r_{\beta}, L\right]=6 \operatorname{Tr}(L) I_{3}\left[r_{\beta}, L\right]
\end{array}\right\} \\
& \left\{\begin{array}{l}
I_{5}\left[q_{\alpha}, L\right]=24\left[3 \operatorname{Tr}(L)+2 \operatorname{Tr}\left(L^{3}\right)\right] I_{2}\left[q_{\alpha}, L\right] \\
I_{5}\left[r_{\beta}, L\right]=\frac{120}{7}\left[3+2 \operatorname{Tr}\left(L^{2}\right)\right] I_{3}\left[r_{\beta}, L\right] \\
I_{5}\left[s_{\gamma}, L\right]=10 \operatorname{Tr}(L) I_{4}\left[s_{\gamma}, L\right]
\end{array}\right\} ; \\
& \left\{\begin{array}{l}
I_{6}\left[q_{\alpha}, L\right]=\frac{360}{7}\left[9+8 \operatorname{Tr}\left(L^{2}\right)+5 \operatorname{Tr}\left(L^{4}\right)\right] I_{2}\left[q_{\alpha}, L\right] \\
I_{6}\left[r_{\beta}, L\right]=\frac{300}{7}\left[9 \operatorname{Tr}(L)+5 \operatorname{Tr}\left(L^{3}\right)\right] I_{3}\left[r_{\beta}, L\right] \\
I_{6}\left[s_{\gamma}, L\right]=\frac{50}{3}\left[8+5 \operatorname{Tr}\left(L^{2}\right)\right] I_{4}\left[s_{\gamma}, L\right] \\
I_{6}\left[t_{\iota}, L\right]=15 \operatorname{Tr}(L) I_{5}\left[t_{\iota}, L\right]
\end{array}\right\} .
\end{aligned}
$$

The reduction formulas displayed in Theorem 8 show that the basic periods are merely those given by equation (73) [which is reassuring]. We also have the following:

COROLlaRY. Let $\left\{M_{k}\right\}_{k=1}^{N}$ be arbitrary elements in $\Gamma$. When evaluated at the origin, the Jacobian matrix of the equi-dimensional mapping $\lambda \rightarrow\left[\operatorname{Im} \operatorname{Tr} \hat{\chi}\left(M_{k}\right)\right]$ is simply: 


$$
J=\frac{1}{(n-1) !}\left[\begin{array}{c}
\operatorname{Im} I_{n}\left(q_{\alpha}, M_{k}\right) \\
-\overline{I m} I_{n}\left(r_{\beta}, M_{k}\right) \\
-\operatorname{Im} I_{n}\left(s_{\gamma}, M_{k}\right) \\
-\overline{\operatorname{Im}} \overline{I_{n}}\left(\bar{t}_{\iota}, \bar{M}_{k}\right) \\
\overline{\operatorname{Im}} \overline{I_{n}}\left(w_{k}, \bar{M}_{k}\right)
\end{array}\right] \quad \text { [with the appropriate truncation]. }
$$

Needless to say, $\lambda$ stands for $\left(\lambda_{\alpha}, \lambda_{\beta}, \ldots\right) \in \mathbf{R}^{N}$.

Observe that $J$ is well defined for any collection of Poincaré series $\left\{q_{\alpha}, r_{\beta}, \ldots\right\} \subseteq V_{n}$, linearly independent or not. [It is understood here that $1 \leqslant \alpha \leqslant 6 g-6,1 \leqslant \beta \leqslant 10 g-10$, etc.

We propose to generalize equation (61) by considering the period matrix $J$. In fact, suppose that $\operatorname{rank}(J)=N$. It follows immediately that $\left\{q_{\alpha}, r_{\beta}, \ldots\right\}$ is a basis for $V_{n}$ (over R). But, now, choose any $h \in\{2,3, \ldots, n\}$. The rows in $J$ corresponding to $\mathbb{Q}_{h}(H, \Gamma)_{\mathbf{R}}$ are linearly independent. For this reason, we can find a subset $\left\{M_{k_{\lambda}}\right\}_{\lambda=1}^{(2 h-1)(2 g-2)}$ such that:

$$
\operatorname{rank}\left[\operatorname{Im} I_{n}\left(\varphi_{j}, M_{k_{\lambda}}\right)\right]=(2 h-1)(2 g-2),
$$

where $\left\{\varphi_{j}\right\}_{j=1}^{(2 h-1)(2 g-2)}$ is any basis for $\mathbb{C}_{h}(H, \Gamma)_{\mathbf{R}}$. Equation (94) can then be regarded as a generalization of Theorem 3 . In view of the reduction formulas in Theorem 8, we also see that:

$$
\operatorname{rank}\left[\operatorname{Im} I_{h}\left(\varphi_{j}, M_{k_{\lambda}}\right)\right]=(2 h-1)(2 g-2) .
$$

A moment's thought shows that the analysis of $J$ is plagued by the same difficulties that were encountered earlier near conclusion (A). In particular, before we can develop a numerical approach based on $J$, it will be necessary to find some way to narrow down the choice of $\left\{M_{k}\right\}_{k=1}^{n}$.

At first sight, it might appear that we have gained nothing by considering $V_{n}$ in place of $\mathbb{Q}_{n}(H, \Gamma)_{\mathbf{R}}$ [especially from a computational point-of-view]. But, in the present case, $N=\operatorname{dim}_{\mathrm{C}}[N(g, n) / S L(n, \mathbf{C})]$. This equation suggests that the correct choice of $M_{k}$ may have something to do with the geometry of $N(g, n) / S L(n, \mathrm{C})$. Cf. conclusion (B).

To clarify matters, we return to equation (90) and observe that $N(g, n) / S L(n, \mathrm{C})$ is nonsingular near the monodromy group $\chi(\Gamma)$ $\bmod S L(n, C)$; cf. [13], [14] and [23, Theorem 2]. In addition, note that the lexicographic correspondence $M \rightarrow \tilde{M}$ [defined after (23)] is still valid for $N(g, n)$; there is no harm in replacing $\pi_{1}(F, \theta)$ by $\Gamma$. Compare: equations (19)-(21). If life is reasonable, there should now exist a set of functions $\left\{\operatorname{Tr}\left(\tilde{M}_{k}\right)\right\}_{k=1}^{N}$ which represent local coordinates [on $N(g, n) / S L(n, \mathrm{C})$ ] near $\chi(\Gamma) \bmod S L(n, \mathrm{C})$.

TheOREM 9 [for $2 \leqslant n \leqslant 6$ ]. Given Poincaré series $\left\{q_{\alpha}, r_{\beta}, \ldots\right\} \subseteq V_{n}$ and elements $\left\{M_{k}\right\} \subseteq \Gamma$. Suppose that $J$ has maximal rank. Then:

(a) the Poincaré series $\left\{q_{\alpha}, r_{\beta}, \ldots\right\}$ form an explicit basis for $V_{n}$;

(b) equations (94) and (95) hold;

(c) the functions $\operatorname{Tr}\left(\tilde{M}_{k}\right)$ are local coordinates near $\chi(\Gamma) \bmod S L(n, \mathbf{C})$. 
Theorem 10 [for $2<n<6$ ]. Given Poincaré series $\left\{q_{\alpha}, r_{\beta}, \ldots\right\} \subseteq V_{n}$ and elements $\left\{M_{h o}: 1<\sigma<(2 h-1)(2 g-2), 2<h<n\right\} \subseteq \Gamma$ such that:

$$
\left\{\begin{array}{c}
\operatorname{rank}\left[\operatorname{Im} I_{2}\left(q_{\alpha}, M_{2 \sigma}\right)\right]=6 g-6 \\
\operatorname{rank}\left[\operatorname{Im} I_{3}\left(r_{\beta}, M_{3 \sigma}\right)\right]=10 g-10 \\
\ldots
\end{array}\right\}
$$

Let $\mathscr{D}$ denote the union of all the elements $M_{h \sigma} ;$ write $\mathscr{Q}^{\lambda}=\left\{M^{\lambda}: M \in \mathscr{D}\right\}$. The hypotheses of Theorem 9 will then hold for an appropriate subset $\left\{M_{k}\right\}_{k=1}^{N}$ of

$$
\cup\left\{\mathscr{Q}^{j}:-\llbracket \frac{n-1}{2} \rrbracket<j<\llbracket \frac{n}{2} \rrbracket, j \neq 0\right\} .
$$

These two theorems form the basis of our computational approach to Poincaré series; cf. Examples 4-6 below.

Theorem 10 is proved by making a detailed study of the period ratios

$$
\frac{I_{n}\left[\varphi, M^{k}\right]}{I_{n}[\varphi, M]} \text { for }\left\{\begin{array}{l}
k \in \mathbf{Z}, M \in \Gamma, M \neq I \\
\varphi \in \mathbb{Q}_{h}(H, \Gamma), 2<h<n
\end{array}\right\}
$$

In the primary case $h=n$, one finds that:

$$
I_{n}\left[\varphi, M^{k}\right]=k\left(\frac{m^{k}-m^{-k}}{m-m^{-1}}\right)^{n-1} I_{n}[\varphi, M],
$$

where $m$ and $m^{-1}$ are the eigenvalues of $M$. Cf. equations (58) and (73). The secondary cases $2<h<n$ can then be treated using Theorem 8. By direct computation, we discover that:

$$
\operatorname{rank}\left[\begin{array}{c}
I_{n}\left(q, M^{j}\right) / I_{n}(q, M) \\
\hdashline I_{n}\left(r, M^{j}\right) / I_{n}(r, M) \\
\hdashline I_{n}\left(s, M^{j}\right) / I_{n}(s, M) \\
--\cdots---
\end{array}\right]=n-1
$$

when $j$ is restricted as in Theorem 10 and $(q, r, s, \ldots) \in \mathbb{Q}_{2} \times \mathbb{Q}_{3} \times \cdots \times$ $\mathbb{Q}_{n}$. Note that the matrix entries are rational functions of $m$ [independent of $q, r, s, \ldots]$.

To complete the proof of Theorem 10, we must check that:

$$
\operatorname{rank}\left[\begin{array}{l}
\operatorname{Im} I_{n}\left(q_{\alpha}, L\right) \\
\hdashline \operatorname{Im} I_{n}\left(r_{\beta}, L\right) \\
\hdashline \operatorname{Im} I_{n}\left(s_{\gamma}, L\right) \\
\hdashline-:--
\end{array}\right]_{L \in \cup \mathscr{D}}=N .
$$

This assertion can be proved by a repeated application of the column operations suggested by (97). Cf. [7, p. 163 (Corollary 3)] and the reduction formulas in Theorem 8. 
Before turning to the examples, we point out that there is a slight extension of Theorem 9 which clarifies the choice of $M_{k}$. For notational simplicity, define:

(98) $\mathscr{T}=\left[\left\{R_{k}\right\}_{k=1}^{N}: \operatorname{Tr}\left(\tilde{R}_{k}\right)\right.$ are local coordinates near $\left.\chi(\Gamma) \bmod S L(n, \mathbf{C})\right]$.

Let $J^{*}$ be the period matrix corresponding to $\left\{L_{k}, q_{\alpha}, r_{\beta}, \ldots\right\}$. Then, under the hypotheses of Theorem 9 ,

$$
\operatorname{rank}\left(J^{*}\right)=N \Leftrightarrow\left\{L_{k}\right\}_{k=1}^{N} \in \mathcal{T} .
$$

By extending some of the arguments found in [18, pp. 240-253], we can easily determine several choices of $\left\{M_{k}\right\}_{k=1}^{N}$ which look like they might belong to $\mathcal{T}$. In view of (99), it is perfectly legitimate to use educated guesses of this type when trying to construct examples in which $\operatorname{rank}(J)=N$.

For convenience, we define:

$$
T_{1}=A_{1}, T_{2}=B_{1} A_{1}^{-1} B_{1}^{-1}, \ldots, T_{2 g-1}=A_{g}, T_{2 g}=B_{g} A_{g}^{-1} B_{g}^{-1} .
$$

Cf. equation (20) and [18, p. 247].

Example 4 ( $g=2$ and $n=3$ ). For generic $\Gamma$, the hypotheses of Theorem 9 are satisfied with:

$$
\begin{aligned}
&\left\{q_{\alpha}\right\}_{\alpha=1}^{6}=\left\{Q_{A_{1}}, Q_{B_{1}}, Q_{A_{2}}, Q_{B_{2}}, Q_{T_{1} T_{2}}, Q_{T_{1} T_{3}}\right\} ; \\
&\left\{r_{\beta}\right\}_{\beta=1}^{10}=\left\{R_{A_{1}}, R_{B_{1}}, R_{A_{2}}, R_{B_{2}}, R_{T_{1} T_{2}}, R_{T_{1} T_{3}}, R_{T_{1} T_{2}^{-1}}, R_{T_{1} T_{3}^{-1}}, R_{A_{2} B_{2}}, R_{A_{2} B_{2}^{-1}}\right\} ; \\
&\left\{M_{k}\right\}_{k=1}^{16}=\left\{A_{1}, A_{2}, B_{1}, B_{2}, T_{1} T_{2} ; T_{1} T_{2}^{-1}, A_{1} B_{1}, T_{1} T_{3}, T_{1} T_{3}^{-1},\right. \\
&\left.A_{2} B_{2}, A_{2} B_{2}^{-1} ; A_{1}^{-1}, A_{2}^{-1}, B_{1}^{-1}, B_{2}^{-1}, T_{1}^{-1} T_{2}^{-1}\right\} .
\end{aligned}
$$

The letters $Q_{L}, R_{L}$ signify relative Poincaré series of weight 2 and 3, respectively.

By using equation (76) and the analog of Theorem 5 , it is very easy to see that $[\operatorname{det}(J)]^{2}$ defines a meromorphic function $\Delta$ on the quotient variety $N(g, 2) / S L(2, \mathrm{C})$. To prove that $\operatorname{det}(J) \neq 0$ for generic $\Gamma$, it is enough to show that $\Delta \neq 0$ on $N(g, 2) / S L(2, \mathrm{C})$. This problem can be settled by a lengthy computation; we find that $\Delta \neq 0$ near the point $\left[X_{\alpha} ; Y_{\beta}\right]$ $\bmod S L(2, \mathrm{C})$, where:

$$
\begin{aligned}
& X_{1}=\left(\begin{array}{cc}
m & 0 \\
0 & m^{-1}
\end{array}\right), \quad Y_{1}=\left(\begin{array}{cc}
\frac{1}{2} & -2 \\
1 & -2
\end{array}\right), \\
& X_{2}=\left(\begin{array}{ll}
1 & 2 \\
1 & 3
\end{array}\right), \quad Y_{2}=\left(\begin{array}{ll}
1 & 1 \\
0 & 1
\end{array}\right), \quad m=\sqrt{2} .
\end{aligned}
$$

[The necessary calculations can all be done by hand.]

EXAmple 5 ( $g=3$ and $n=3$ ). For generic $\Gamma$, the hypotheses of Theorem 9 are satisfied with: 


$$
\begin{gathered}
\left\{q_{\alpha}\right\}_{\alpha=1}^{12}=\left\{Q_{A_{1}}, Q_{A_{2}}, Q_{A_{3}}, Q_{B_{1}}, Q_{B_{2}}, Q_{B_{3}}, Q_{T_{1} T_{2}}, Q_{T_{2} T_{3}},\right. \\
\left.Q_{T_{3} T_{4}}, Q_{T_{1} T_{3}}, Q_{T_{2} T_{4}}, Q_{T_{3} T_{5}}\right\} ; \\
\left\{r_{\beta}\right\}_{\beta=1}^{20}=\left\{R_{A_{1}}, R_{A_{2}}, R_{A_{3}}, R_{B_{1}}, R_{B_{2}}, R_{B_{3}}, R_{T_{1} T_{2}}, R_{T_{2} T_{3}}, R_{T_{3} T_{4}},\right. \\
R_{T_{1} T_{3}}, R_{T_{2} T_{4}}, R_{T_{3} T_{5}}, R_{T_{1} T_{2}^{-1}}, R_{T_{2} T_{3}^{-1}}, R_{T_{3} T_{4}^{-1}} \\
\left.R_{T_{1} T_{3}^{-1}}, R_{T_{2} T_{4}^{-1}}, R_{T_{3} T_{5}^{-1}}, R_{A_{3} B_{3}}, R_{A_{3} B_{3}^{-1}}\right\} ; \\
\left\{M_{k}\right\}_{k=1}^{32}=\left\{\begin{array}{c}
A_{1}, A_{2}, A_{3}, B_{1}, B_{2}, B_{3}, T_{1} T_{2}, T_{2} T_{3}, T_{3} T_{4} ; T_{1} T_{2}^{-1}, T_{2} T_{3}^{-1} \\
T_{3} T_{4}^{-1}, A_{1} B_{1}, A_{1} B_{2}, A_{2} B_{2}, T_{1} T_{3}, T_{2} T_{4}, T_{3} T_{5}, T_{1} T_{3}^{-1}, \\
\\
T_{2} T_{4}^{-1}, T_{3} T_{5}^{-1}, A_{3} B_{3}, A_{3} B_{3}^{-1} ; A_{1}^{-1}, A_{2}^{-1}, A_{3}^{-1}, \\
B_{1}^{-1}, B_{2}^{-1}, B_{3}^{-1}, \\
\left.T_{1}^{-1} T_{2}^{-1}, T_{2}^{-1} T_{3}^{-1}, T_{3}^{-1} T_{4}^{-1}\right\}
\end{array}\right.
\end{gathered}
$$

Note that $\Delta$ can be defined as in Example 4. By means of a very lengthy computation, it can be proved that $\Delta \neq 0$ near the point $\left[X_{\alpha} ; Y_{\beta}\right]$ $\bmod \operatorname{SL}(2, \mathrm{C})$, where:

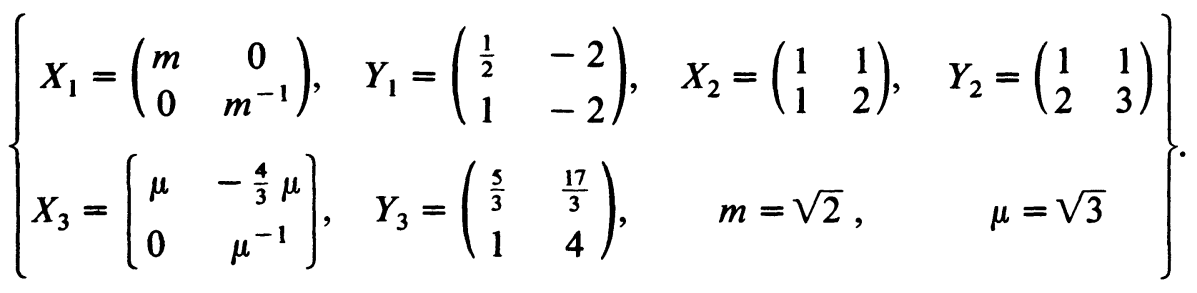

In this connection, we remark that:

[A] The problem ultimately reduces to showing that a certain $32 \times 32$ matrix $J_{0}$ is nonsingular [at the point $\left(X_{\alpha} ; Y_{\beta}\right) \bmod S L(2, \mathrm{C})$ ]. The matrices $J$ and $J_{0}$ are very closely related.

[B] It took 14 days to finish this project: 6 days to (theoretically) determine the entries in $J_{0}, 5$ days to compute their values numerically, 3 days to evaluate $\operatorname{rank}\left(J_{0}\right)$. An SR-52 programmable calculator was used wherever possible.

[C] After a few reductions, $J_{0}$ transforms into a matrix having rational entries. The denominators turn out to be integers of the form $2^{a} 3^{b}$. At this point, it is convenient to reduce everything modulo 11 . Since this reduction leads to a nonsingular matrix over $\mathbf{Z}_{11}$, we conclude that $\operatorname{rank}\left(J_{0}\right)=32$. [On the other hand, we observe that $\mathbf{Z}_{5}$ does not work.]

EXAMPLE $6(g=2$ and $n=4)$. For generic $\Gamma$, the hypotheses of Theorem 10 are satisfied when: 


$$
\begin{aligned}
& \left\{q_{\alpha}\right\}_{\alpha=1}^{6}=\left\{Q_{A_{1}}, Q_{B_{1}}, Q_{A_{2}}, Q_{B_{2}}, Q_{T_{1} T_{2}}, Q_{T_{1} T_{3}}\right\} ; \\
& \left\{r_{\beta}\right\}_{\beta=1}^{10}=\left\{R_{A_{1}}, R_{B_{1}}, R_{A_{2}}, R_{B_{2}}, R_{T_{1} T_{2}}, R_{T_{1} T_{2}^{-1}}, R_{T_{1} T_{3}}, R_{T_{1} T_{3}^{-1}}, R_{A_{2} B_{2}}, R_{A_{2} B_{2}^{-1}}\right\} ; \\
& \left\{S_{\gamma}\right\}_{\gamma=1}^{14}=\left\{S_{A_{1}}, S_{B_{1}}, S_{A_{2}}, S_{B_{2}}, S_{T_{1} T_{2}^{-1}}, S_{T_{1} T_{2}^{2}}, S_{T_{1} T_{3}}, S_{T_{1} T_{3}^{-1}}, S_{T_{1} T_{3}^{2}}\right. \\
& \left.S_{A_{2} B_{2}}, S_{A_{2} B_{2}^{-1}}, S_{A_{2} B_{2}^{2}}, S_{A_{1} B_{1}}, S_{A_{1} B_{2}}\right\} ; \\
& \left\{M_{2 \sigma}\right\}_{\sigma=1}^{6}=\left\{A_{1}, B_{1}, A_{2}, B_{2}, T_{1} T_{2}, T_{1} T_{3}\right\} ; \\
& \left\{M_{3 \sigma}\right\}_{\sigma=1}^{10}=\left\{A_{1}, B_{1}, A_{2}, B_{2}, T_{1} T_{2}, T_{1} T_{2}^{-1}, T_{1} T_{3}, T_{1} T_{3}^{-1}, A_{2} B_{2}, A_{2} B_{2}^{-1}\right\} ; \\
& \left\{M_{4 \sigma}\right\}_{\sigma=1}^{14}=\left\{A_{1}, B_{1}, A_{2}, B_{2}, T_{1} T_{2}^{-1}, T_{1} T_{2}^{2}, T_{1} T_{3}, T_{1} T_{3}^{-1}, T_{1} T_{3}^{2},\right. \\
& \left.A_{2}, A_{2} B_{2}^{-1}, A_{2} B_{2}^{2}, A_{1} B_{1}, A_{1} B_{2}\right\} .
\end{aligned}
$$

The letters $Q_{L}, R_{L}, S_{L}$ signify relative Poincaré series of weight $2,3,4$.

Let $J_{2}, J_{3}, J_{4}$ denote the period matrices which appear in Theorem 10 [when $n=4$ ]. As in Example 4, we easily see that $\left[\operatorname{det}\left(J_{k}\right)\right]^{2} \operatorname{defines}$ a meromorphic function $\Delta_{k}$ on the quotient variety $N(g, 2) / S L(2, \mathrm{C})$. In order to check that $\Delta_{k} \neq 0$, it will be enough to show that certain matrices $\tilde{J}_{2}, \tilde{J}_{3}, \tilde{J}_{4}$ have maximal rank. [Needless to say, the matrices $J_{k}$ and $\tilde{J}_{k}$ are very closely related.] The associated computations can be carried through successfully for the point $\left[X_{\alpha} ; Y_{\beta}\right] \bmod S L(2, \mathrm{C})$, where:

$$
\begin{aligned}
& X_{1}=\left(\begin{array}{cc}
m & 0 \\
0 & m^{-1}
\end{array}\right), \quad Y_{1}=\left(\begin{array}{cc}
\frac{1}{2} & -2 \\
1 & -2
\end{array}\right), \\
& X_{2}=\left(\begin{array}{ll}
1 & 2 \\
1 & 3
\end{array}\right), \quad Y_{2}=\left(\begin{array}{ll}
1 & 1 \\
0 & 1
\end{array}\right), \quad m=\sqrt{2} .
\end{aligned}
$$

The only serious trouble occurs when $k=4$. In this case, it is convenient to use the methods explained at the end of Example 5. In particular, the denominators are powers of 2 and a reduction modulo 11 works. Cf. remark [C]. Counting the time spent on bad choices of $s_{\gamma}$ and $M_{4 \sigma}$, the analysis of $\Delta_{4}$ took me approximately two weeks (using the SR-52).

REMARK. For safety's sake, the computations in Examples 4-6 were all double-checked.

We shall now examine a more "theoretical" approach to the generalization of Theorem 3. [Recall method (B) near equation (77).] To save space, it will be assumed that the reader has some familiarity with Eichler cohomology. Cf. [9] and [34, Chapter 5]. It is customary to let $\Pi_{m}$ denote the vector space of all polynomials (over $\mathbf{C}$ ) with degree $\leqslant m$.

Fix any basis $\left\{q_{\alpha}, r_{\beta}, s_{\gamma}, \ldots\right\}$ for $V_{n}$; observe that these differentials are not assumed to be Poincare series. Let the corresponding accessory parameters be $\left\{\lambda_{\alpha}, \lambda_{\beta}, \lambda_{\gamma}, \ldots\right\}$ as in equation (85). For notational convenience, we select any one of the accessory parameters [say $\lambda$ ] and suppose that $\lambda$ corresponds to $\varphi \in \mathbb{Q}_{k}(H, \Gamma)$.

The following result can then be proved by applying Theorem 7 near the trivial equation $u^{(n)}=0$. The normalization is understood to be that given by equations (86) and (89). 
THEOREM 11 [for $2<n \leqslant 6$ ]. When evaluated at the trivial equation, we find that

$$
\frac{\partial \hat{\chi}(L)}{\partial \lambda}=A \chi(L)-\chi(L) A-\frac{1}{(n-1) !} \int_{z_{0}}^{L z_{0}} \varphi(z) \Re_{n k}(z) d z \cdot \chi(L),
$$

where:

(i) $A$ is an $n \times n$ matrix with trace zero whose entries depend solely on (n, $\left.\varphi, z_{0}\right)$;

(ii) $\mathfrak{N}_{n k}(z)$ is an $n \times n$ matrix whose entries are monomials depending solely on $(k, n)$. n]:

The matrices $\Re_{n j}(z)$ satisfy the following additional properties [ for $2<j<$

(a) $\mathfrak{T}_{n n}(z)=X(z) Y(z)^{t}=\mathfrak{N}(z)$ as in equation (71);

(b) $\mathfrak{N}_{n j}(T z)=T^{\prime}(z)^{j-1} \cdot \chi(T) \mathfrak{N}_{n j}(z) \chi\left(T^{-1}\right)$ for $T \in S L(2, \mathrm{C})$;

(c) $\operatorname{deg} \mathfrak{T}_{n j}(z)=2 j-2$;

(d) $\operatorname{Tr}\left[\Re_{n j}(z)\right]=0$;

(e) $\operatorname{Tr}\left[\mathfrak{R}_{n j}(z) \mathfrak{R}_{n k}(w)\right]=c_{n k} \delta_{j k}(z-w)^{2 k-2}$, where $\delta_{j k}$ is the Kronecker delta and

$$
\left\{\begin{array}{l}
c_{22}=-1 \\
c_{33}=+1, c_{32}=-9 \\
c_{44}=-1, c_{43}=24, c_{42}=-648 / 5 \\
c_{55}=+1, c_{54}=-50, c_{53}=4800 / 7, c_{52}=-2880 \\
c_{66}=-1, c_{65}=90, c_{64}=-2500, c_{63}=180000 / 7, c_{62}=-648000 / 7
\end{array}\right\} .
$$

Assertions (a)-(e) were verified by direct computation. This method of proof becomes very unpleasant when $n \geqslant 7$. For this reason, it would be useful to have an alternate approach to these formulas. It seems fairly obvious that such an approach can be found by appropriate use of group representations and/or invariant theory. For the latter, we refer to [60, pp. 175-199] and [70]. We note that similar remarks can be made regarding Theorems 6-8. On the other hand, it is not at all clear how to attack equation (97) for large $n$.

Continuing onward, let

$$
p: N(g, n) \rightarrow N(g, n) / S L(n, \mathrm{C})
$$

denote the obvious projection map. It is known that $p$ defines an analytic fiber bundle near those points $\left[X_{\alpha} ; Y_{\beta}\right]$ which correspond to irreducible representations of $\Gamma$; cf. [13], [14]. In particular, this will be true for a small neighborhood $\Re$ surrounding the point $\chi(\Gamma) \in N(g, n)$.

FACT 1. One can always determine local coordinates $\left[\tau_{1}, \ldots, \tau_{N}\right]$ on $N(g, n) / S L(n, \mathrm{C})$ so that:

(a) $\tau_{1}=\cdots=\tau_{N}=0$ corresponds to $\chi(\Gamma) \bmod S L(n, \mathbf{C})$;

(b) $\left[\tau_{1}, \ldots, \tau_{N}\right] \in \mathbf{R}^{N}$ iff $p^{-1}\left(\tau_{1}, \ldots, \tau_{N}\right)$ intersects $\Re \cap S L(n, \mathbf{R})^{2 g}$ nontrivially. 
For convenience, we use $\tau=\left(\tau_{1}, \ldots, \tau_{N}\right)$ to represent points of $N(g, n) / S L(n, C)$ near $\chi(\Gamma) \bmod S L(n, C)$. [Observe that $\tau$ will be a point in $\mathbf{C}^{N}$ near the origin.]

FACT 2. There exists a holomorphic family $\left[X_{\alpha}(\tau) ; Y_{\beta}(\tau)\right]$ of points in $\mathscr{T}$ such that:

(a) $\left[X_{\alpha}(0) ; Y_{\beta}(0)\right]=\chi(\Gamma)$;

(b) $p\left[X_{\alpha}(\tau) ; Y_{\beta}(\tau)\right]=\tau$;

(c) the point $\left[X_{\alpha}(\tau) ; Y_{\beta}(\tau)\right]$ belongs to $\mathscr{R} \cap S L(n, \mathbf{R})^{2 g}$ iff $\tau \in \mathbf{R}^{N}$.

By use of the lexicographic correspondence $M \rightarrow \tilde{M}$, we can now define a holomorphic mapping $\tilde{L}(\tau)$ for any $L \in \Gamma$. The generators obviously correspond to $\tilde{A}_{\alpha}(\tau)=X_{\alpha}(\tau)$ and $\tilde{B}_{\beta}(\tau)=Y_{\beta}(\tau)$. For small values of $|\lambda|$, we let $D E(\lambda)$ represent the $n$th order equation whose accessory parameters are all zero except for $\lambda$. Cf. equations (82) and (85). The holomorphic mappings $\hat{L}(\lambda)$ and $\tilde{L}(\lambda)$ can then be defined by writing:

$$
\lambda \rightarrow[\text { normalized } m \text {-group of } D E(\lambda)] \rightarrow \hat{\chi}(L)=\hat{L}(\lambda)
$$

[cf. Theorems 7 and 11];

$$
\lambda \rightarrow[\text { normalized } m \text {-group of } D E(\lambda)] \stackrel{p}{\rightarrow}[\tau] \stackrel{i}{\rightarrow} \tilde{L}(\tau)=\tilde{L}(\lambda) \text {. }
$$

We emphasize that these mappings are defined only for small values of $\lambda$.

Since $\hat{L}(0)=\tilde{L}(0)$, it follows that there is a uniquely determined matrix $M(\lambda) \in S L\left(n\right.$, C) such that: (i) $M(0)=I$; (ii) $\tilde{L}(\lambda)=M(\lambda) \hat{L}(\lambda) M(\lambda)^{-1}$ for all $L \in \Gamma$; (iii) $M(\lambda)$ depends holomorphically on $\lambda$. Note that $\hat{L}(\lambda)$ and $\tilde{L}(\lambda)$ are homomorphisms with respect to $L$.

FACT 3. Suppose that $2<j<n$. Then,

$$
\mathscr{P}_{L j}(x)=\operatorname{Tr}\left[\frac{d \tilde{L}(\lambda)^{-1}}{d \lambda} \cdot \chi(L) \mathscr{M}_{m j}(x)\right]
$$

defines a cocycle in $H^{1}\left[\Gamma, \Pi_{2 j-2}\right]$. It is understood here that $x$ is a real variable and that the $\lambda$-derivative is evaluated at $\{\lambda=0\}$.

Using Theorem 11, it is trivial to differentiate the relation $\tilde{L}(\lambda)=$ $M(\lambda) \hat{L}(\lambda) M(\lambda)^{-1}$. The end-result can then be substituted into Fact 3. [Notice that $M^{\prime}(0)$ has trace zero.]

THEOREM 12 [for $2<n<6$ ]. Introduce the accessory parameter $\lambda$ as above, and suppose that $\lambda$ corresponds to $\varphi \in \mathbb{Q}_{k}(H, \Gamma)$. Then,

$$
\begin{aligned}
\mathscr{P}_{L}(x) & =\operatorname{Tr}\left[\frac{d \tilde{L}(\lambda)^{-1}}{d \lambda} \chi(L) \Re_{n j}(x)\right] \\
& =P_{j}(x)-P_{j}(L x) L^{\prime}(x)^{1-j}+E \delta_{j k} \int_{L^{-1} z_{0}}^{z_{0}} \varphi(z)(z-x)^{2 k-2} d z
\end{aligned}
$$

where: (a) $2<j<n$; (b) $x$ is a real variable; (c) the $\lambda$-derivative is evaluated at $\{\lambda=0\}$; (d) $E$ is a nonzero real constant dependent solely on $(k, n)$; (e) $\boldsymbol{P}_{j}(x) \in \Pi_{2 j-2} ;(f) P_{j}(x)=\operatorname{Tr}\left[Q \Re_{n j}(x)\right]$ for some $n \times n$ matrix $Q$ having zero 
trace. Once $\left[X_{\alpha}(\tau) ; Y_{\beta}(\tau)\right]$ is chosen, the matrix $Q$ depends solely on $\left(n, \varphi, z_{0}\right)$.

To interpret Theorem 12 in terms of Eichler cohomology, we consider the Eichler integral

$$
F(z)=\int_{z_{0}}^{z}(z-t)^{2 k-2} \varphi(t) d t
$$

as in [9] and observe that

$$
F(L z) L^{\prime}(z)^{1-k}-F(z)=\int_{L^{-1} z_{0}}^{z_{0}}(z-u)^{2 k-2} \varphi(u) d u .
$$

The period cocycle for $F$ will therefore be given by:

$$
Q_{\varphi L}(x)=\int_{L^{-1} z_{0}}^{z_{0}}(u-x)^{2 k-2} \varphi(u) d u \in \Pi_{2 k-2} \quad \text { for } L \in \Gamma .
$$

As usual, $x$ denotes a real variable.

Corollary [to Theorem 12]. Fix $\lambda$ and $\varphi$ as above. For $2 \leqslant j \leqslant n$, we find that: $\mathscr{P}_{L j}(x)=[$ coboundary $]+E \delta_{j k} Q_{\varphi L}(x)$.

If $p(x) \in \Pi_{2 j-2}$, then $\operatorname{Re} p(x)$ and $\operatorname{Im} p(x)$ have obvious meanings. In particular, it is easy to see that $\operatorname{Im} Q_{\varphi L}(x)$ defines a cocycle in $H^{1}\left[\Gamma, \Pi_{2 k-2}\right]$.

THEOREM 13 [for $2 \leqslant k<\infty$ ]. Assume that $\varphi \in \mathbb{Q}_{k}(H, \Gamma)$. Then, $\operatorname{Im} Q_{\varphi L}(x)$ is a coboundary iff $\varphi(z) \equiv 0$.

For the proof, we refer to [34, pp. 215-219] and [64a, pp. 278-285]. Theorem 13 plays a crucial role in the derivation of the next theorem.

To clarify matters, suppose that $\left\{M_{k}\right\}_{k=1}^{N} \in \mathcal{T}$ as in equation (98). Modulo a trivial translation, we can now use $t_{k}=\operatorname{Tr}\left(\tilde{M}_{k}\right)$ in place of $\tau_{k}$. The holomorphic mappings $\tilde{L}(t)$ can then be defined, and we obtain

$$
\tilde{L}(t)=F_{L}\left(t_{1}, \ldots, t_{N}\right)
$$

for appropriate functions $F_{L}$ near the point $\chi(\Gamma) \bmod S L(n, \mathrm{C})$. In view of Fact $2, F_{L}$ is real for $\left(t_{1}, \ldots, t_{N}\right) \in \mathbf{R}^{N}$. [The functions $F_{L}$ should be regarded as "known" quantities; a little investigation shows that they are algebraic in nature.]

Observe that

$$
\tilde{L}(\lambda)=\left\{\begin{array}{l}
F_{L}\left[\operatorname{Tr} \tilde{M}_{1}(\lambda), \ldots, \operatorname{Tr} \tilde{M}_{N}(\lambda)\right] \\
F_{L}\left[\operatorname{Tr} \hat{M}_{1}(\lambda), \ldots, \operatorname{Tr} \hat{M}_{N}(\lambda)\right]
\end{array}\right\}
$$

Accordingly, when the derivatives are evaluated at $\left[u^{(n)}=0\right]$, we find that:

$$
\begin{aligned}
\operatorname{Tr}\left[\frac{d \tilde{L}(\lambda)^{-1}}{d \lambda} \chi(L) \mathscr{R}_{n j}(x)\right] & =\sum_{h=1}^{N} \operatorname{Tr}\left[\frac{\partial F_{L^{-1}}}{\partial t_{h}} \chi(L) \Re_{n j}(x)\right] \frac{d \operatorname{Tr} \hat{M}_{h}(\lambda)}{d \lambda} \\
& =\sum_{h=1}^{N} \operatorname{Tr}\left[\frac{\partial F_{L^{-1}}}{\partial t_{h}} \chi(L) \Re_{n j}(x)\right] \frac{I_{n}\left[\varphi, M_{h}\right]}{(n-1) !},
\end{aligned}
$$

via equation (90). It is easy to see that 


$$
\mathscr{P}_{L h j}(x)=\operatorname{Tr}\left[\frac{\partial F_{L^{-1}}}{\partial t_{h}} \chi(L) \Re_{n j}(x)\right]
$$

defines a cocycle in $H^{1}\left[\Gamma, \Pi_{2 j-2}\right]$ with real coefficients; we emphasize that this partial derivative is evaluated at $\chi(\Gamma) \bmod S L(n, \mathrm{C})$. The earlier equations will now yield:

$$
\mathscr{P}_{L j}(x)=\frac{1}{(n-1) !} \sum_{h=1}^{N} \mathscr{P}_{L h j}(x) I_{n}\left[\varphi, M_{h}\right] .
$$

Applying the corollary, we discover that:

(111) [coboundary] $+E \delta_{j k} Q_{\varphi L}(x)=\frac{1}{(n-1) !} \sum_{h=1}^{N} \mathcal{P}_{L h j}(x) I_{n}\left[\varphi, M_{h}\right]$;

(112) [coboundary $]+E \delta_{j k} \operatorname{Im} Q_{\varphi L}(x)=\frac{1}{(n-1) !} \sum_{h=1}^{N} \mathscr{P}_{L h j}(x) \operatorname{Im} I_{n}\left[\varphi, M_{h}\right]$.

Since (112) holds for each basis vector $\varphi \in\left\{q_{\alpha}, r_{\beta}, s_{\gamma}, \ldots\right\}$, there will be a linear relationship between $J$ and the imaginary parts of the Eichler periods $Q_{\varphi L}(x)$. Cf. equation (93). By investigating the appropriate linear combinations of (112) using Theorem 13, we conclude that $J$ has maximal rank.

TheOREM 14 [for $2 \leqslant n \leqslant 6$ ]. Let $\left\{q_{\alpha}, r_{\beta}, s_{\gamma}, \ldots\right\}$ be any basis for $V_{n}$. The period matrix $J$ has maximal rank if and only if $\left\{M_{k}\right\}_{k=1}^{N} \in \mathcal{T}$. For the definition of $\mathcal{T}$, consult equation (98).

In some sense, Theorem 14 is the "heart of the matter." Observe that the "only if" follows from Theorem 9(c); cf. the completeness theorems in $\$ 5$.

COROLlaRY [for $2 \leqslant n \leqslant 6$ ]. Suppose that $R$ belongs to $\Re_{n}$ and that its poles are located at hyperbolic fixpoints. If $\mathcal{T}$ is nonempty, then the identical vanishing of $\theta[z, R] \in \mathbb{Q}_{n}(D, \Gamma)$ can be decided in finite terms. Cf. equations (48)-(49).

As far as I can determine, the structure of $\mathcal{T}$ has never been carefully investigated when $n \geqslant 3$. Cf. [4], [40], [58]. Needless to say, it seems very probable that $\mathcal{T}$ is nonvoid for all $\Gamma$.

In the case of generic $\Gamma$, it should be possible to construct explicit examples of $\left\{M_{k}\right\}_{k=1}^{N} \in \mathcal{T}$. Cf. Examples $4-6$ and Theorem $9(\mathrm{c})$ for $(g, n)=(2,3)$, $(3,3),(2,4)$.

Before closing, it may be useful to mention several areas which deserve further investigation:

(I) eliminate the restriction $2 \leqslant n \leqslant 6$ using group representations and/or invariant theory;

(II) give a careful description of $\mathcal{T}$ in the case of arbitrary $(g, n)$;

(III) prove analogous results [about Poincaré series] in the case of bordered Riemann surfaces;

(IV) find some number-theoretical applications for these techniques by considering congruence subgroups;

(V) try to formulate some results for the so-called zeta-Fuchsian series [54]; 
(VI) try to attack $\theta[z, R]$ over $H$ instead of $D$;

(VII) study the periods $I_{n}[\varphi, L]$ in connection with nonanalytic automorphic forms; cf. [12, pp. 293-294], [25, p. 473], [36], [37], [38].

We hope to return to problems (III)-(V) in a later paper.

\section{REFERENCES}

1. L. Ahlfors, Lectures on quasi-conformal mappings, Van Nostrand, New York, 1966.

2. L. Ahlfors and L. Sario, Riemann surfaces, Princeton Univ. Press, Princeton, N.J., 1960.

3. P. Appell, E. Goursat, and P. Fatou, Theorie des fonctions algebriques, vol. 2, GauthierVillars, Paris, 1930.

4. M. Artin, On Azumaya algebras and finite dimensional representations of rings, J. Algebra 11 (1969), 532-563.

5. L. Bers, An approximation theorem, J. Analyse Math. 14 (1965), 1-4.

6. _ On spaces of Riemann surfaces with nodes, Bull. Amer. Math. Soc. 80 (1974), 1219-1222.

7. G. Birkhoff and S. Mac Lane, A survey of modern algebra, 3rd ed., MacMillan, New York, 1965.

8. M. Dehn, Transformation der Kurven auf zweiseitigen Flächen, Math. Ann. 72 (1912), 413-421.

9. M. Eichler, Eine Verallgemeinerung der Abelschen Integrale, Math. Z. 67 (1957), 267-298.

10. R. Fricke and F. Klein, Vorlesungen über die Theorie der automorphen Funktionen, vol. 1, Teubner, Leipzig, 1897.

11. 1912.

. Vorlesungen über die Theorie der automorphen Funktionen, vol. 2, Teubner, Leipzig,

12. I. M. Gelfand, M. I. Graev, and I. I. Pyatetskii-Shapiro, Representation theory and automorphic functions, Saunders, New York, 1969.

13. R. Gunning, Analytic structures on the space of flat vector bundles over a compact Riemann surface, Several Complex Variables II, Lecture Notes in Math., vol. 185, Springer-Verlag, Berlin and New York, 1971, pp. 47-62.

14. __ Lectures on vector bundles over Riemann surfaces, Princeton Univ. Press, Princeton, N.J., 1967, especially Chapter 9.

15. H. Hancock, Theory of elliptic functions, Dover, New York, 1958.

16. G. A. Hedlund, On the metrical transitivity of the geodesics on closed surfaces of constant negative curvature, Ann. of Math. 35 (1934), 787-808.

17. D. A. Hejhal, Monodromy groups and linearly polymorphic functions, Acta Math. 135 (1975), $1-55$.

18. (1976), 215-264. , The variational theory of linearly polymorphic functions, J. Analyse Math. 30

19. , Sur les paramètres accessoires pour l'uniformisation de Schottky, C. R. Acad. Sci. Paris Sér. A-B 279 (1974), 713-716.

20. __ Quelques remarques à propos des séries de Poincaré sur les groupes de Schottky, $\mathrm{C}$. R. Acad. Sci. Paris Sér. A-B 280 (1975), 341-344.

21. __ Sur les paramètres accessoires pour l'uniformisation fuchsienne, C. R. Acad. Sci. Paris Sér. A-B 282 (1976), 403-406.

22. __ Sur les séries de Poincaré des groupes fuchsiens, C. R. Acad. Sci. Paris Sér. A-B 284 (1977), 607-610.

23. __ Monodromy groups for higher-order differential equations, Bull. Amer. Math. Soc. 81 (1975), 590-592.

24. , On Schottky and Teichmüller spaces, Advances in Math. 15 (1975), 133-156.

25. 441-482.

26. K. Hensel and G. Landsberg, Theorie der algebraischen Funktionen einer Variabeln, Teubner, Leipzig, 1902.

27. D. Hilbert, Gesammelte Abhandlungen, vol. 3, Springer-Verlag, Berlin, 1935. 
28. F. Klein, Neue Beiträge zur Riemann'schen Funktionentheorie, Math. Ann. 21 (1883), 141-218.

29. __ Gesammelte mathematische Abhandlungen, vol. 3, Springer-Verlag, Berlin, 1923.

30. U Über lineare Differentialgleichungen der zweiten Ordnung, Göttingen, 1894 (handwritten lecture notes), especially pp. 97, 116-118, 459, 462-464, 477-478, 499-514.

31. __ Vorlesungen über die Hypergeometrische Funktion, Springer-Verlag, Berlin, 1933.

32. P. Koebe, Über die Uniformisierung beliebiger analytischer Kurven, Göttingen Nachr. (1907), 191-210; (1907),.633-669; (1908), 337-358; (1909), 324-361.

33. __ Über die Uniformisierung beliebiger analytischer Kurven, J. Reine Angew. Math. 138 (1910), 192-253; 139 (1911), 251-292.

34. I. Kra, Automorphic forms and Kleinian groups, Benjamin, New York, 1972.

35. W. Magnus, A. Karrass, and D. Solitar, Combinatorial group theory, 2nd ed., Dover, New York, 1976, especially pp. 24-29.

36. Ju. I. Manin, Explicit formulas for the eigenvalues of Hecke operators, Acta Arith. 24 (1973), 239-249.

37. __ Periods of parabolic forms and p-adic Hecke series, Math. USSR-Sb. 21 (1973), 371-393.

38. __ Parabolic points and zeta-functions of modular curves, Math. USSR-Izv. 6 (1972), 19-64.

39. M. Morse, A fundamental class of geodesics on any closed surface of genus greater than one, Trans. Amer. Math. Soc. 26 (1924), 25-60.

40. D. Mumford, Geometric invariant theory, Springer-Verlag, Berlin and New York, 1965.

41. P. J. Myrberg, Über die numerische Ausführung der Uniformisierung, Acta Soc. Sci. Fenn. 48 (1920), no. 7, 1-53.

42. __ Sur les fonctions automorphes, Ann. École Norm. Sup. 68 (1951), 383-424.

43. - Uber automorphe Funktionen, Proceedings of the International Congress of Mathematicians 1954, vol. 3, pp. 118-126.

44. J. Nielsen, Untersuchungen zur Topologie der geschlossenen zweiseitigen Flächen, Acta Math. S0 (1927), 189-358.

45. H. Petersson, Die linearen Relationen zwischen ganzen Poincaréschen Reihen von reeller Dimension zur Modulgruppe, Abh. Math. Sem. Univ. Hamburg 12 (1938), 415-472.

46. __ Zur analytischen Theorie der Grenzkreisgruppen. V, Math. Z. 44 (1939), 127-155.

47. __ Einheitliche Begründung der Vollständigkeitssätze für die Poincaréschen Reihen,

Abh. Math. Sem. Univ. Hamburg 14 (1941), 22-60.

48. _ U Uber Weierstrasspunkte und die expliziten Darstellungen der automorphen Formen von reeller Dimension, Math. Z. 52 (1950), 32-59.

49 , Automorphe Formen als metrische Invarianten, Math. Nachr. 1 (1948), 158-212, 218-257.

50. __ Die Systematik der Abelschen Differentiale in der Grenzkreisuniformisierung, Ann. Acad. Sci. Fenn. 276 (1960), 74pp .

51. H. Poincaré, Mémoire sur les fonctions fuchsiennes, Acta Math. 1 (1882), 193-294.

52. __ Sur une théorème de la théorie générale des fonctions, Bull. Soc. Math. France 11 (1883), 112-125.

53. __ Sur les groupes des équations linéaires, Acta Math. 4 (1884), 201-312.

54. __ Mémoire sur les fonctions zétafuchsiennes, Acta Math. 5 (1884), 209-278.

55. __ Sur les invariants arithmétiques, J. Reine Angew. Math. 129 (1905), 89-150.

56. __ Sur l'uniformisation des fonctions analytiques, Acta Math. 31 (1907), 1-64.

57. __ Fonctions modulaires et fonctions fuchsiennes, Ann. Fac. Sci. Toulouse 3 (1912),

125-149.

58. C. Procesi, The invariants of $n \times n$ matrices, Bull. Amer. Math. Soc. 82 (1976), 891-892.

59. B. Riemann, Gesammelte mathematische Werke, 2nd ed., Teubner, Leipzig, 1892, especially pp. $301,379,440,445$.

60. L. Schlesinger, Handbuch der Theorie der linearen Differentialgleichungen, vol. 2, Teubner, Leipzig, 1897.

61. __ Vorlesungen über lineare Differentialgleichungen, Teubner, Leipzig, 1908.

62. F. Schottky, Über die conforme Abbildung mehrfach zusammenhängender ebener Flächen, J. Reine Angew. Math. 83 (1877), 300-351. 
63. Über eine specielle Function, welche bei einer bestimmten linearen Transformation ihres Arguments unverändert bleibt, J. Reine Angew. Math. 101 (1887), 227-272.

64. H. A. Schwarz, Gesammelte mathematische Abhandlungen, vol. 2, Springer-Verlag, Berlin, 1890 , especially pp. $65-83,350-355$.

64a. G. Shimura, On Dirichlet series and abelian varieties attached to automorphic forms, Ann. of Math. (2) 76 (1962), 237-294.

65. C. L. Siegel, Topics in complex function theory, vol. 1, Wiley, New York, 1969.

66. O. Teichmüller, Bestimmung der extremalen quasikonformen Abbildungen bei geschlossenen orientierten Riemannschen Flächen, Abh. Preuss. Akad. Wiss. (Berlin) 4 (1943), 1-42.

67. M. Tsuji, Behnke-Stein's theorem on the existence of a basic differential on a compact Riemann surface with boundary curves, Comment. Math. Univ. St. Paul. 8 (1960), 45-51.

68. J. Walsh, Interpolation and approximation, 3rd ed. Amer. Math. Soc. Colloq. Publ., no. 20, Amer. Math. Soc., Providence, R.I., 1960.

69. H. Weyl, The concept of a Riemann surface, 3rd ed., Addison-Wesley, Reading, Mass., 1955.

70. E. J. Wilczynski, Projective differential geometry of curves and ruled surfaces, Teubner, Leipzig, 1906.

Department of Mathematics, Columbia University, New York, New York 10027 